Canadian

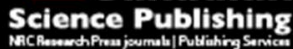

Canadian Geotechnical Journal Revue canadienne de géotechnique

\title{
Distributed Temperature Sensing applied during diaphragm wall construction
}

\begin{tabular}{|c|c|}
\hline Journal: & Canadian Geotechnical Journal \\
\hline Manuscript ID & cgj-2014-0522.R1 \\
\hline Manuscript Type: & Article \\
\hline Date Submitted by the Author: & 17-Dec-2015 \\
\hline Complete List of Authors: & $\begin{array}{l}\text { Spruit, Rodriaan; TU Delft, Geo Engineering; Municipality of Rotterdam, } \\
\text { Engineering } \\
\text { van Tol, Frits; TU Delft, Geo Engineering } \\
\text { Broere, Wout; Delft University of Technology, GeoEngineering Section } \\
\text { Doornenbal, Pieter; Deltares, } \\
\text { Hopman, Victor; Deltares, }\end{array}$ \\
\hline Keyword: & $\begin{array}{l}\text { Distributed Temperature Sensing, DTS, diaphragm wall, joint, quality } \\
\text { control }\end{array}$ \\
\hline
\end{tabular}




\title{
Distributed Temperature Sensing applied during diaphragm wall construction
}

\author{
Rodriaan Spruit ${ }^{1,2}$, Frits van Tol ${ }^{1,3}$, Wout Broere ${ }^{1}$, Pieter Doornenbal ${ }^{3}$, Victor Hopman ${ }^{3}$ \\ 1: Department of Civil Engineering, Delft University of Technology, P.O. box 5, 2600 AA, \\ Delft, Netherlands \\ 2: Project Management \& Engineering, Municipality of Rotterdam, Rotterdam, Netherlands \\ 3: Deltares, Utrecht/Delft, Netherlands \\ Corresponding author: Rodriaan Spruit (e-mail: r.spruit@tudelft.nl)
}

\begin{abstract}
Distributed Temperature Sensing (DTS) can be used to monitor the production process of diaphragm walls. DTS is able to differentiate between already present and fresh bentonite suspensions during refreshing of the bentonite slurry and excavation bentonite remaining in the trench can be observed. During concrete casting, DTS is able to differentiate between bentonite suspension and concrete. As a result, the continuity of the casting process and the arrival of good grade concrete at crucial locations in the trench can be monitored.

Tests conducted on laboratory models provided reference information for interpretation of field data. Field experiences have shown the benefits of the DTS tests and the predictive value of the reference measurements. Finally, the results are compared with CSL measurements at the same location.
\end{abstract}

Key words: Distributed Temperature Sensing, DTS, diaphragm wall, joint, quality control

\section{Resumé}

Détection Distribué du Température (DDT) peut être utilisé pour surveiller le processus de production des parois moulées. DDT est capable de différencier entre suspensions de bentonite déjà présentes dans la tranchée et bentonite fraîches au cours rafraîchissant de la suspension bentonite. Lors de la coulée du béton, DDT est capable de différencier entre la suspension de bentonite et de béton. En conséquence, la continuité du processus de coulée et l'arrivée d'un béton de bonne qualité à des endroits cruciaux dans la tranchée peuvent être surveillés.

Les tests effectués sur des modèles de laboratoire ont fourni des informations de référence pour l'interprétation des données de terrain. Expériences de terrain ont montré les avantages des tests DDT et la valeur prédictive des mesures de référence. Enfin, les résultats sont comparés avec les mesures d'auscultation sonique au même endroit.

Mots clés: Détection Distribué du Température, paroi moulée, joint, contrôle de qualité

\section{Introduction}


Diaphragm walls ( $D$-walls) are frequently used for deep underground constructions in densely populated areas because of their high strength and stiffness in combination with relatively silent and vibration-free installation as compared to a sheet piled wall. Notwithstanding the extensive experience in design and construction of D-walls, quality control for both the water tightness and retaining functions has proven to be difficult, as evidenced by calamities during construction works in the Netherlands and Belgium (Van Tol et al. 2010; Berkelaar 2011; Van Tol and Korff 2012). Poor quality or even absence of concrete in the joints between the diaphragm wall panels is seen as the primary cause of these failures (Van Tol et al. 2010). Other examples of below grade performance have been reported in Boston (Poletto and Tamaro 2011), Cologne (Sieler et al. 2012) and Taipei (Hwang et al. 2007).

Methods to detect anomalies in diaphragm walls are studied, particularly in the area around the joints between the panels, prior to excavation of the building pit enclosed by the diaphragm walls. Crosshole Sonic Logging (CSL) testing has since been verified in the laboratory and successfully implemented in several projects in the Netherlands (Spruit et al. 2014). Distributed Temperature Sensing (DTS) is envisioned as another way of detecting anomalies.

In the DTS technique, a glass fiber which acts as a linear optical sensor is interrogated with a DTS device, delivering a continuous temperature profile along the sensor. In other engineering fields like hydrology (Selker et al. 2006, Tyler et al. 2009) and petroleum exploration (Brown and Tiwari 2010), DTS is often used to monitor fluid transportation and distribution for which a spatial resolution in the order of meters is required. However, for monitoring concrete casting a much higher spatial accuracy is required because the expected height differences within the trench are in the order of a few decimeters, making a centimeter order of magnitude resolution required for detecting irregularities in the casting process. Experiments during the construction of an underground parking facility in Rotterdam (Spruit et al. 2011) show promising results for monitoring concrete flow using DTS during diaphragm wall production (Doornenbal et al. 2011; Spruit et al. 2011).

This paper will focus on the verification tests of DTS in the laboratory and in field setups.

\section{Hypothesis}

During the construction process of a diaphragm wall, bentonite and concrete with different temperatures replace each other in the different construction phases. During the de-sanding operation fresh bentonite replaces the excavation bentonite, and the temperature of the fresh bentonite will differ from that of the bentonite in the trench. During concrete casting, the concrete replacing the bentonite will once again differ in temperature from the bentonite. In most cases the concrete will have a higher temperature than the bentonite in the trench. Detailed and continuous temperature measurements near the joints during de-sanding and concrete casting would enable monitoring of the presence of concrete in a joint between two diaphragm walls.

Finally, during curing the concrete will heat up and a locally lower temperature could indicate an area with sub-optimal concrete properties.

With Distributed Temperature Sensing (DTS) the required temperature measurements should be possible and practical. The principle of DTS measurements for quality control during concrete curing has been applied since the late nineteen-nineties (Thevenaz et al, 1998), especially in large arched dams and other large volume concrete structures. 
More recently other applications have become common, especially in ground water monitoring (Selker et al. 2006, Tyler et al. 2009). However, the effectiveness of tracking the concrete casting of diaphragm walls or of the bentonite refreshing operation has not been published before.

\section{Measurement principle}

The DTS measurement uses glass fibers that are installed at critical locations in the diaphragm wall, such as the joint to the adjacent panel, around the water stop or behind areas with a very dense rebar grid that might obstruct the concrete flowing through. Useful installation options are:

- lowering the sensor in the bentonite with a weight attached to the sensor end;

- attaching the sensor to the rubber water stop before installation of the stop end;

- attaching the sensor to the rebar cage.

Sensors lowered in the trench with a weight attached to the sensor end should always be close to the trench wall allowing the diverging concrete flow to push the sensors to the trench wall, locking their position.

All three methods have been tested in the field and show no significant differences in temperature recording behavior.

With a DTS device, the fiber is interrogated, offering a continuous temperature profile of the fiber, essentially making the glass fiber a continuous linear temperature sensor. In the rest of the paper the glass fiber will be called sensor.

In this study, DTS measurements based upon the Raman scatter principle were used. In these measurements, a monochromatic laser pulse is fed into the sensor. The vast majority of the light will be transmitted through the sensor. A small portion of light interacts inelastically with the electrons in the sensor and generates light at two frequencies symmetrical about the injected light frequency (Figure 1). The reflected light band with lower frequency is referred to as 'Stokes', and the reflected light band with higher frequency than injected is referred to as 'Anti-Stokes'. With increasing local temperature, more electrons end up in the high energy state, thus increasing the anti-Stokes/Stokes ratio. Contrary to the Brillouin scatter, which is sensitive to both strain and temperature, the Raman scatter is not influenced by local strain in the sensor (Selker et al. 2006). As the speed of light is known, it can be determined at what position in the sensor the reflected spectrum that is recorded in time was generated. This type of DTS measurements therefore belongs to the Optical Time Domain Reflectometry (OTDR) family.

Figure 1: Raman scatter principle (after Selker et al. 2006)

By analyzing the ratio of anti-Stokes over Stokes as a function of time, the local temperature of the sensor can be derived. To obtain a good signal to noise ratio for the measurements, multiple measurements need to be stacked (Selker et al. 2006). A longer measurement time will therefor lead to more accurate determination of the local temperature in the sensor, provided that the temperature is not changing during acquisition. Applied in a diaphragm wall during concrete casting, the local temperatures will change continuously, making a compromise between temperature accuracy and short acquisition time necessary, as 
indicated in the discussion paragraph. The DTS device will produce per sensor position the local temperature along the full length of the sensor. The spatial resolution (generally every measurement is averaged over $1 \mathrm{~m}$ ) is independent of the optical fiber and depends on the measurement equipment used.

\section{Laboratory measurements}

First, the behaviour of the DTS sensor which was intended for the field experiments has been tested in laboratory conditions. For these tests a ruggedized optical fiber (ACE-TKF CTC 8xMM) connected to a Sensornet Oryx DTS (Sensornet, 2012) has been selected. This sensor contains 8 MultiMode fibers in a gel-filled plastic tube protected with Kevlar fibers covered with a plastic outer liner, as shown in Figure 2. The external diameter of the sensor is approximately $7 \mathrm{~mm}$.

The following parameters have been explored because they are not generally provided by the manufacturer: the response time of a sudden temperature change, the pressure dependency, and the accuracy of the spatial resolution.

Response time

A single ended sensor cable has been conditioned for at least five minutes in a container with water of about 20 degrees Celsius. Immediately after completion of a measurement cycle the sensor cable is submerged in a container with warm water (around 50 degrees Celsius) and several subsequent measurements of one minute are recorded to construct the asymptote of the temperature adjustment. Figure 3 shows the accommodation speed for this specific sensor. The ACE-TKF CTC 8xMM cable needs between 70 and 100 seconds to fully adapt to the surrounding temperature when immersed in water.

Figure 2: Cross section of the ACE-TKF CTC 8xMM cable

Figure 3: Temperature adaptation in time

Because the hot water was cooling down during the test, a relative temperature drop has been used instead of an absolute temperature drop so that several measurements (in this case 5 immersion tests) can be combined.

Although this ruggedized sensor has shown a good survival rate in the field, the thick liner causes a slow equalizing of the temperature in the sensor. For fast and accurate temperature acquisition, a sensor with a thinner liner should be considered. The increased vulnerability could be compensated by installing more sensors than strictly needed (redundancy). As sensors with a thin liner are generally cheaper than ruggedized sensors, the redundancy concept applied to thin lined sensors can be more cost effective than a monitoring setup with ruggedized sensors.

\section{Pressure dependency}

Because of the intended application in a diaphragm wall, the sensor could be exposed to external pressures ranging from 0 to 14 bar (considering a maximum depth of the diaphragm 
wall of about $60 \mathrm{~m}$ ). In the field tests in Delft, the diaphragm walls reached a depth of $20 \mathrm{~m}$, exposing the lowest parts of the sensors to a pressure of about 5 bar.

To check the pressure dependency, a test fiber has been installed in a pressure tank. The tank was $90 \%$ filled with water at 20 degrees Celsius to act as a temperature buffer and the air void above the water was pressurized to 6 bar. The temperature readings from the sensor did not change during this test. Temperature drift of this sensor cable due to pressure change is therefor considered negligible within the expected in-situ pressure range.

During the field tests in Rotterdam, where a maximum depth of $42 \mathrm{~m}$ was reached (equivalent to about 10 bar pressure), no temperature drift in the vertical profile was noticed, which seems to substantiate the expected pressure independency of this sensor system.

\section{Spatial accuracy and resolution}

In a casting form instrumented with optical DTS sensors, the possibilities of DTS to detect a clay inclusion with varying thickness have been explored previously, as reported by Doornenbal et. al. (2011). It was concluded that the thickness of a clay layer separating the DTS sensor from the cast concrete could be derived from the measurement data.

In order to verify if fresh bentonite arrives at critical locations in the panel during slurry refreshing, or if good grade concrete arrives at critical locations in the panel during the concrete casting phase, it is necessary to determine the response curve of the sensor and DTS device in a situation with two fluids at different temperatures. This has been done using two containers with water of different temperature placed next to each other. Several meters of the same fiber have been placed in each container, as sketched in Figure 4. As the water level in the containers was almost to the top level, the transition zone of the sensor between the two containers (initially around at position $8.389 \mathrm{~m}$ along the length of the fiber) was less than $0,05 \mathrm{~m}$ in length. During continuous recording, the sensor was kept stationary for ten minutes (ten temperature recordings) after which the transition zone was shifted $0.2 \mathrm{~m}$. This was repeated until the transition zone had shifted $0.8 \mathrm{~m}$ in total (final position of transition zone at $7.589 \mathrm{~m}$ ).

Figure 4: Test setup for determining the response curve

Figure 5: Relative temperature difference for 3 measurement positions (in meters relative to the DTS device) in the linear sensor near the transition zone during the response curve test

Figure 5 illustrates how the temperature readings in front and behind the transition zone are affected relative to the temperature difference between the hot and cold containers. Each of the 3 measurement positions show 5 temperature plateaus, corresponding to the 5 positions, $0.2 \mathrm{~m}$ apart, of the transition zone. It can also be concluded that sensor position $8.159 \mathrm{~m}$ is slightly off the center of the test as can be seen from the 3rd measurements (second $0.2 \mathrm{~m}$ shift) sequence (see Figure 4 ) which are at 0.6 relative temperature instead of 0.5 relative temperature. 
The average of each set of ten temperature readings has been used to determine Figure 6 . After the fourth shift, it seems that no equilibrium has been reached at measurement position $7.144 \mathrm{~m}$. However, this does not show in Figure 6, confirming that 10 minutes is a wellchosen interpolation period for determining the response curve. During in-situ measurements, shorter interpolation times can be accepted, as indicated in the discussion section.

Figure 6: Response curve for Sensornet Oryx DTS and single ended ACE-TKF CTC 8xMM fiber based upon ten averaged recordings with acquisition time of 1 minute (model as line and measurements as dots, relative temperature $0.5=50 \%$ of $\delta \mathrm{T}$, where $\delta \mathrm{T}=|\mathrm{T} 1-\mathrm{T} 2|$ and $\mathrm{T} 1=$ constant temperature before transition zone, $\mathrm{T} 2=$ constant temperature after transition zone)

The best fit through the relative temperature readings is given by

$$
\frac{T}{T_{1}-T_{0}}=-0,1927 \sqsubset x^{2}+0,6164 \sqsubset x
$$

In which:

$\mathrm{T}=$ measured temperature

$\mathrm{T}_{1}=$ temperature of medium 1

$\mathrm{T}_{0}=$ temperature of medium 0

$\mathrm{x}=$ sensor position relative to position of interface between media 1 and 0

For convenient implementation in a spreadsheet program, equation 1 has been replaced by a best fitting $5^{\text {th }}$ order equation, starting at the start of the transition zone, which is for the Oryx DTS device $1,5 \mathrm{~m}$ in front of the position of the interface between de media (Figure 7).

Figure 7: Simplified response curve for Sensornet Oryx DTS and single ended ACE-TKF CTC 8xMM fiber using a $5^{\text {th }}$ order equation. The interface between the two media is located at position $1,5 \mathrm{~m}$.

The $5^{\text {th }}$ order equation shown in Figure 7 , is applied only in the transition zone using the Boolean functions of the spreadsheet program.

For a three phase system, each interface is simulated with its own equation. The combined result is the numerical addition of the equation (2) for each transition zone. In the part describing the transition between medium 2 and 3, all sub-numbers should be one integer higher.

$\mathrm{T}_{\mathrm{s}}=$ simulated temperature at position $\mathrm{O}$ (degrees $\mathrm{C}$ )

$\mathrm{O}=$ observation point $(\mathrm{m}$ to sensor start)

$\mathrm{I}_{1}=$ interface between medium 1 and medium 2 ( $\mathrm{m}$ to sensor start)

$\mathrm{I}_{2}=$ interface between medium 2 and medium 3 ( $\mathrm{m}$ to sensor start)

$\mathrm{T}_{1}$ =temperature of medium 1

$\mathrm{T}_{2}=$ temperature of medium 2

$\mathrm{T}_{3}$ =temperature of medium 3 


$$
\begin{aligned}
& \mathrm{a}=0.0243 \\
& \mathrm{~b}=-0.1819 \\
& \mathrm{c}=0.3888 \\
& \mathrm{~d}=-0.1126 \\
& \mathrm{e}=0.1208 \\
& \mathrm{f}=-0.0034 \\
& \text { For } \\
& I_{1}-O \geq 1.5 \\
& \Rightarrow T_{s}=T_{1} \\
& 1.5>I_{1}-O>-1.5 \\
& \Rightarrow T s=T_{1}+\left(T_{2}-T_{1}\right) *\left(a *\left(O-I_{1}+1.5\right)^{5}+b *\left(O-I_{1}+1.5\right)^{4}+c *\left(O-I_{1}+1.5\right)^{3}+d *\left(O-I_{1}+1.5\right)^{2}+e *(O-\right. \\
& (2) \\
& -1,5 \geq I_{1}-O \\
& \Rightarrow T_{s}=T_{2}
\end{aligned}
$$

Note that this response curve is specific for the Sensornet Oryx DTS device. If another DTS recorder is used, a different curve could be applicable. Following the procedure described above, the response curve should be determined if the manufacturer does not provide such information.

Although we generally would expect a DTS device with more measurements per meter to have a steeper response curve, theoretically it could have a less steep response curve. Without determining the actual response curve, it is not proven that a DTS device with, for example, 5 measurements per meter is 5 times more accurate than a device that only measures 1 temperature per meter.

Because the acquisition time only improves the reliability (and reduces the bandwidth) of the temperature readings, it is expected that the general shape of the response curve remains the same when the acquisition time of the temperature recording is reduced, but that the individual points on the curve will show more variation.

The response curve for the Sensornet Oryx DTS shows that the local temperature between $1.5 \mathrm{~m}$ before and after the observation point influences the resulting measurement at the observation point.

As long as we recognize that we are dealing with only two media, each with a specific temperature, this poses no trouble for the interpretation. On the contrary, using the response curve we are able to locate the actual interface between the two media much more accurately than is suggested by the spatial resolution of $1 \mathrm{~m}$ that is stated in the device specifications. However, if the total sensor length in a medium is less than $3 \mathrm{~m}$ and we do not know the exact dimensions of the medium, we will be unable to determine the exact temperature of the sub-3 m length of sensor. If we know the temperature by means of another measurement, we will be able to determine the actual length using the characteristics from Figure 6. Consequently, if a reliable calibration of the temperature measured with the sensor is needed, a calibration coil with at least $6 \mathrm{~m}$ of sensor in a 
controlled temperature zone is recommended. Generally, if only the position of the interface of two media is required, a relative temperature reading is sufficient.

Using the response curve from Figure 6, the known sensor positions during the test and the temperature in both the warm and cold water containers, the measured absolute temperatures from Figure 5 have been simulated. These simulations are compared with the measured temperatures in Figure 8. Measured temperatures are depicted by dashed lines and simulated temperatures by solid lines. To illustrate how the temperature of the hot container (sensor position 10.188) dropped during the test and the container with cold water (sensor position 6.129) warmed up during the test, these sensor positions are added. Note that sensor position 10.188 , which was initially completely in the hot container, was slightly affected by the cold container at more than a meter distance, which is coherent with Figure 6 . To determine the temperature in the hot container, the readings at sensor position 12.217 have been used.

Figure 8: Measured (dashed) and simulated (continuous) temperature response curves for sensor positions near the transition zone

\section{Field measurements}

In 2010 the DTS sensors were applied in-situ for the first time in $42 \mathrm{~m}$ deep diaphragm walls for an underground parking underneath Kruisplein in Rotterdam. It was anticipated that the heat generated during curing of the concrete would primarily render useful information. The same ruggedized ACE-TKF CTC 8xMM sensor as tested in the lab was used. The slow response was not considered to be a problem as the temperature build-up during concrete curing is much slower than the accommodation speed of the sensor. However, the measurements started just before concrete casting.

The sensors were located as shown in the cross-section in Figure 9.

The sensors at positions 1 and 2 were installed in the primary panel (attached to the rubber water stop and to the rebar cage respectively). The secondary panel does not allow for sensor position 1 on this joint as the stop end does not facilitate the required space for the sensor cable. In the opposing joint of the secondary panel, sensors at positions 1 and 2 were applied. All sensors were positioned vertically with the sensor end at the bottom of the excavated trench (no loops or other intricate shapes). At sensor position 3 , the sensor was lowered into the trench with a weight at the sensor end.

Figure 9: Cross section with DTS sensors relative to joint and rebar cages as applied during the field tests in Rotterdam

The measurements were surprisingly illustrative for the rising concrete level in the trench during casting, although it seemed probable that for tracking concrete level changes in the trench the response time could influence the measurements. It was therefor considered worthwhile to further investigate the accuracy of concrete level determination using DTS.

The temperature measurements during curing gave less information than expected, because the soil properties around the diaphragm wall seemed to govern the temperature in the wall. The ambient soil temperature at the site is on average 12 degrees $C$ which seems to have little correlation with the peak curing temperature. 
The peak curing temperature (Figure 10) for all sensor positions was highest in the peat layers, while within the clay layers intermediate temperatures were recorded and the lowest temperatures were recorded where the diaphragm wall was embedded in sand layers. This correlates well with thermal conductivity prediction models that indicate an increasing thermal conductivity with increasing density (Farouki 1981). Even within one layer (especially the sand layer) different temperatures were encountered, indicating that detection of anomalies in diaphragm walls based on peak curing temperature is difficult at best.

Figure 10: Peak temperature profile for sensor position 2 (Figure 9) during concrete curing with CPT and boring

In 2011 DTS profiles were recorded in the railway tunnel project through the city of Delft in the Netherlands. The D-wall panels reached a depth of $25 \mathrm{~m}$ below surface level.

The expected development of subsequent temperature profiles recorded during concrete casting is indicated in Figure 11. The spacing of the temperature profiles will be defined by the recording interval and the vertical velocity of the concrete casting front.

Figure 11: Expected temperature profile sequence during concrete casting

In Figure 12 subsequent temperature profiles are shown for the center of the panel (close to the tremie pipe, see Figure 19). The interval between the profiles was four minutes because the DTS device was interrogating 4 fibers in sequence with an interpolation time of one minute for each fiber. Due to the large number of measurements in time, the overall impression of such a graph is confusing.

At the positions indicated with arrows two or more temperature profiles overlap. Depending on the number of overlapping profiles, this means a multitude of four minutes of stagnation during concrete casting. This could be caused by cutting the tremie pipe or changing the concrete truck at the tremie. Longer and therefore more hazardous discontinuations in the concrete flow would of course show up in the sequence of temperature profiles more predominantly as they would include a lot of overlapping profiles. The arrows are placed halfway between the temperatures of concrete and bentonite, as seems logical from the response curve from Figure 6.

Figure 12: Subsequent temperature profiles with an interval of 4 minutes in the center of the panel during concrete casting, arrows indicating overlapping temperature profiles of subsequent measurements

Around the same time as the stagnations in Figure 12 were found, similar stagnations (overlapping temperature recordings) occur in Figure 13. The occurrence of these stagnations generally shows good depth correlation between Figure 12 and Figure 13. The sequence of temperature profiles as indicated in Figure 12 and Figure 13 is too dense with information for straight forward interpretation. It seems that the concrete casting front will be trackable with such a sequence if properly interpreted. The temperature profiles then need to be converted to a depth dependent concrete level graph (Figure 22). Sometimes it is not 
clear where to interpret the level of the interface between concrete and bentonite, as is illustrated with Figure 13. The extra wiggle in the graphs around 15.5 to 16 degrees $\mathrm{C}$ does not correspond to the response curve for a single interface between two media. The extra wiggle seems to indicate a layer of relatively constant intermediate temperature, possibly consisting of a mixture of concrete and bentonite. It might be possible to simulate this using a three phase model with two superposed response curves (Figure 15). A simulation of the temperature response could offer more accurate determination of the interface between (high grade) concrete and bentonite (or low grade concrete).

Figure 13: Subsequent temperature profiles with an interval of 4 minutes in the joint area during concrete casting, arrows indicating overlapping temperature profiles of subsequent measurements

The DTS measurements have also been performed during the slurry refreshing operation (Figure 14). The effectiveness of replacing the slurry in the trench (16.2 degrees $C$ ) with freshly mixed slurry (12.6 degrees $\mathrm{C}$ ) (from left to right in the graph) could be determined just as clearly as the concrete casting. The even spacing between the temperature profiles in Figure 14 indicates a constant slurry refreshing speed, which is in accordance with the constant pumping rate of the immersed raw water pump at the bottom of the trench and the synchronized addition of fresh slurry at the top. The rising temperature between 3 and $15 \mathrm{~m}$ below surface level is caused by the still warm adjacent panel, heating up the fresh slurry after entry in the trench. As the slurry in the trench and the surrounding soil have a temperature in the order of 12 degrees $\mathrm{C}$, higher temperatures, especially if they are recorded in the sensor next to a previously cast panel, are probably caused by the still hot previously cast panel.

Figure 14: Subsequent temperature profiles with an interval of 4 minutes in the joint area during slurry refreshing

From the laboratory tests and the field test, it seems possible to determine the actual concrete level using DTS much more accurately than expected regarding the manufacturers' $1 \mathrm{~m}$ spatial resolution.

\section{Correlation with manual concrete level measurements}

To verify the accuracy of the concrete levels determined with the DTS profiles, a comparison has been made with manual concrete level measurements of the same panel.

Using the response curve as shown in Figure 6, each temperature profile from Figure 13 has been simulated. To obtain a good fit with the recorded temperature profiles, a three phase (concrete, mixed material, bentonite) system has been simulated using 2 superimposed response curves.

Figure 15 shows a measured and simulated temperature profile to illustrate the simulated response of a three phase system. 
Figure 15: Measured and simulated temperature profiles in the joint at 19-1-2011 15:58:15. Correlation between measured and simulated graphs $=0.999$ from 10.5 to $20.5 \mathrm{~m}$ below surface level

During the simulation process initially the temperatures for the bentonite, concrete and intermediate layer and the levels for the separation interfaces are assumed. The temperatures can be derived from the relatively constant temperatures in the graph above and below the interface, the interface levels are assumed from the steepest parts of the graph. With these assumptions and the response curve (Figure 6), for each sensor position, the expected temperature response is calculated. If plotted together with the recorded temperatures at exactly the same sensor positions, the simulation can be validated. During the optimization phase of the simulation, the temperatures of the media and the positions of the interfaces are iteratively varied to obtain a visually optimal fit with the measured temperature curve. The simulated graph shown in Figure 15 is the result of a medium (bentonite) with a temperature of 12.5 degrees $C$ from surface level to $14.6 \mathrm{~m}$ below surface level, the second ( $3.2 \mathrm{~m}$ thick) layer of medium between $14.6 \mathrm{~m}$ below surface level till 17.8 $\mathrm{m}$ below surface level with a temperature of 15.9 degrees $C$ and below that the third layer (concrete) with a temperature of 18.6 degrees $\mathrm{C}$. To illustrate the simulation process, a simulated graph with the interface between bentonite and the mixed material $0.5 \mathrm{~m}$ too high and the interface between the mixed material and concrete $0.5 \mathrm{~m}$ too low is shown in Figure 16. The shape of the simulated graph in Figure 16 is correct, but the intermediate step at 16 $\mathrm{m}$ below surface level is too wide compared to the measured curve.

Figure 16: Measured and simulated temperature profiles in the joint at 19-1-2011 15:58:15, upper interface $0.5 \mathrm{~m}$ too high, lower interface $0.5 \mathrm{~m}$ too low. Correlation between measured and simulated graphs $=0.984$ from 10.5 to $20.5 \mathrm{~m}$ below surface level

Between 0 and $8 \mathrm{~m}$ below surface level the temperature of the bentonite mixture was slightly higher because the sensor was positioned in the joint next to the still-warm panel that had been cast 4 days before. The constant temperature between 15.5 and $16.5 \mathrm{~m}$ below surface level indicates a layer with constant temperature. From the simulation shown in Figure 15 it can be concluded that this intermediate layer is $3.2 \mathrm{~m}$ thick (from $14.6 \mathrm{~m}$ till $17.8 \mathrm{~m}$ below surface level) and has an average temperature of 15.9 degrees Celsius.

After simulating all recorded temperature profiles, it has been noticed that to obtain a correctly fitting simulated temperature profile, the position of the interface between two materials has to be accurate to 0.05 to $0.10 \mathrm{~m}$. This suggests that the position of the interface between the two materials can be determined with an accuracy of 0.05 to $0.10 \mathrm{~m}$. This applies for Sensornet Oryx DTS measurements with 1 minute acquisition time per measurement. Due to this relatively long acquisition time and the adaptation time of the sensor, the interface position will be shifting during acquisition, causing a loss in spatial accuracy. A total combined latency of about one minute will cause a delay of one minute in depth recording. This corresponds to a 0.05 to $0.1 \mathrm{~m}$ lower perceived concrete level considering a concrete cast duration of four hours (Figure 22) for a $20 \mathrm{~m}$ deep diaphragm wall panel.

The acquisition time of the DTS device and the latency of the sensor should therefore be shortened if possible while still maintaining acceptable temperature accuracy and 
ruggedness. Figure 17 shows that for relatively short sensors (during the tests the sensors were always less than $150 \mathrm{~m}$ long), the acquisition time does not significantly affect the temperature resolution. For reliable simulation of the temperature response, the temperature difference between the media above and below the interface should preferably be an order of magnitude higher than the temperature resolution.

Figure 17: Temperature resolution Oryx DTS as a function of sensor length (after Sensornet 2012)

Similar to Figure 15, all temperature profiles recorded in the joint and in the center of the diaphragm wall panel were analyzed.

In the center of the panel, close to the tremie pipe, only a two phase system was encountered as illustrated by the temperature measurements and simulations in Figure 18. When comparing Figure 15 and Figure 18, we notice that different temperatures have been found for concrete and bentonite at these different locations. This could partly be caused by cooling of the concrete during horizontal transportation from the tremie pipe to the joint and by variation of the bentonite temperature close to the tremie pipe. On the other hand, DTS measurements based upon Raman scatter (Tyler et al. 2009) do not offer absolute temperatures. Due to slight signal loss in an optical connector for example, the absolute values of the temperature profile can shift.

If we assume the concrete temperature in the joint to be the correct value at 18.6 degrees Celsius and shift the profile in the center of the panel accordingly, we find a bentonite temperature of 14 degrees Celsius which is much closer to the 12.5 degrees Celsius we encountered in the joint. However, the absolute value of the temperature profiles is not significant for determining the location of an interface between materials. If absolute temperature is required, all sensors should run through a temperature-controlled or isolated calibration box for at least $6 \mathrm{~m}$ sensor length as discussed above.

Figure 18: Measured and simulated temperature profiles in the center of the panel

The simulated temperature profiles provide a time sequence of concrete-bentonite interface levels for the center of the panel and a time sequence of concrete-mixed material and mixed material-bentonite interface levels for the joint area. Figure 22 plots these sequences together with the manual depth registrations that were recorded by the contractor against time.

The concrete levels in the center of the panel derived from the simulations (solid line, Figure 22) show a good correlation with the manual recordings (square dots Figure 22). Between the fifth and sixth manual recordings a stagnation of the concrete casting of 12 minutes went unnoticed by the manual concrete levelling, but is clearly noticeable in the concrete levels derived from the temperature profiles. In the joint, the top of the mixed material with intermediate temperature is rising at almost the same speed as the concrete level in the center of the panel. The stagnation of the concrete casting is also visible in the joint area. 
Concrete, with comparable temperature as the concrete in the center of the panel, is observed in the joint area on average $3 \mathrm{~m}$ below the level in the center of the panel. The height of the zone of mixed material gradually increases during the concrete casting. This is understandable, as mixed material will accumulate on top of the concrete in the joint area while it is pushed upwards and towards the joint by the concrete flowing from the center to the panel sides. This also explains why the top of the mixed material zone exceeds the level of the concrete towards the end of the concrete casting period. The top of the concrete in the joint never reaches the top of the panel. This corresponds with general experience with diaphragm walls: the upper meters close to a joint generally contain more contamination with bentonite and poor quality concrete than at lower levels. In this case, according to the levels derived from the temperature profiles, the upper $3 \mathrm{~m}$ of joint is expected to be of poor quality. This was in accordance with CSL measurements (Spruit et al. 2014) and observations on site.

When examining the upper $5 \mathrm{~m}$ of the CSL logs of this specific joint (Figure 20), we encounter a quickly deteriorating signal in the joint (straight (1-2 and 3-4) and diagonal (1-3 and 2-4) joint crossings) at $3 \mathrm{~m}$ below the top of the panel. The CSL logs parallel to the joint (which are located $0.4 \mathrm{~m}$ from the joint) show the same signal deterioration, but at $1.9 \mathrm{~m}$ below the top of the panel.

Figure 19: Position of CSL logs, manual depth recordings and DTS sensors in the panel

Figure 20: CSL logs, loss of signal indicating poor concrete

As the CSL logs parallel to the joint are located $0.4 \mathrm{~m}$ from the joint, we could estimate the slope of the concrete - mixed material interface using the level where deterioration of the signal starts and the position of the CSL logs in the panel. The DTS profiles also provide concrete level information. If the DTS and CSL interpretations are combined, this leads to the concrete boundaries as suggested in Figure 21.

Figure 21: Interpretation of CSL and DTS concrete levels (side view of panel)

Figure 22: Concrete levels recorded in the center and joint of a panel

The correlation with the manual concrete level measurements has shown that the DTS concrete level measurements are in the same order of accuracy. The levels derived from the temperature profiles are more objective and far more frequent than manual recordings. The estimated 0.05 to 0.1 lower perceived concrete levels, based upon DTS sensor and device latency, seem insignificant compared to the accuracy of the manual depth recordings (Figure 22). The manually recorded levels depend on subjectively sensed resistance of the dropped weight onto the concrete and are operator dependent. If a zone of mixed material is present on top of the concrete, this could be mistaken for concrete. Manual recordings are generally 
performed after each truckload of concrete and as a result offer only a few measurements over time. Note that the manual depth registration and the top concrete (solid line) from Figure 22 were registered near the center of the panel (see Figure 19). Both dashed lines were recorded in the joint to the next panel.

The estimated accuracy of the DTS measurements in this case study is $5-10 \mathrm{~cm}$. An accuracy of about $2-5 \mathrm{~cm}$ is probably achievable if the acquisition time of the DTS is reduced to 15 seconds instead of the 60 seconds used here. To reach that accuracy, it is also necessary that the sensors have a liner that is as thin as possible to avoid retarding the temperature measurements. With such accuracy, otherwise difficult to monitor differences, for example the small differences in the concrete level between inside and outside the rebar cage, could be monitored.

\section{Discussion}

The DTS level measurements offer a number of advantages over other concrete level measurements during D-wall casting:

- the sensor cables are relatively low-cost

- the required space for the sensor cable is almost nil, making it possible to measure for example the concrete level between the rebar cage and the trench wall

- several sensor cables can be connected to 1 measurement device, making simultaneous concrete level measurement at several positions in one trench relatively easy

- the sensor cable could be integrated within the water stop that is often used in the joints between panels, providing detection of concrete at the most decisive location for verifying water tightness. The width of the rubber strip also provides separation from the previously cast panel, thus improving the concrete level resolution and reducing the influence of the temperature of the adjacent panel.

- the sensor cable can easily be attached to the rebar cage

- the sensor has negligible influence on the concrete flow process

- vulnerability of the sensor cable is much less of a problem than expected (only $5 \%$ of the ACE-TKF CTC 8xMM sensors failed during the field tests)

- excellent recording of the slurry refreshing and concrete casting process is possible

Disadvantages of the method are:

- optical sensors are vulnerable, especially at the optical connectors where dust and/or moisture can interfere with the measurements

- in a daily operation of diaphragm wall production the sensor cables would be easily damaged if no special care is taken to prevent stepping on the sensor cables or of the sensor cables being squeezed between rebar cage and trench wall etc.

- DTS equipment is still rather expensive and not yet optimized for this specific application

Considering the above mentioned pros and cons, this measurement technique is at this moment most suitable for laboratory circumstances or field test environments intended for (further) understanding of bentonite and concrete flow during diaphragm wall production. DTS could also be useful in specific project situations, such as when a complex and dense rebar cage with possible flow obstruction needs to be verified before serial production. If, in time, a simple-to-operate DTS device specifically designed for this application is available, the concrete level measurement using DTS could become a standard quality control tool for D-wall production. With DTS it will be possible to check proper slurry 
refreshing, allowing for additional cleaning of the trench by brushing the joints and rerefreshing if stagnation or irregularities during refreshing are encountered.

During concrete casting, DTS will offer the possibility to monitor the slope of the casting front, differences between concrete level in- and outside the rebar cage and casting interruptions. The latest generation of DTS devices promises an even higher spatial accuracy, possibly making the concrete level measurement even more accurate than obtained during the tests described in this paper. This should be determined first with response curve measurements as described above.

\section{Conclusions}

DTS measurements can be used to monitor the production of diaphragm walls. During the slurry refreshing operation the replacement of excavation bentonite by freshly mixed bentonite can be monitored. If stagnation during refreshing is encountered, additional cleaning of the trench by methods such as brushing the joints and re-refreshing could be considered. During concrete casting DTS offers the possibility to record stagnation and to verify if good quality concrete arrives along the perimeter of the trench. In the joint, the detected arrival of good quality concrete ensures a high probability of a watertight joint. The optical sensor that is used for DTS might be integrated in the water stop that is often applied in joints between diaphragm wall panels. Other successful installation possibilities include lowering of the sensor using a weight attached to the sensor end or attaching the sensor to the rebar cage. 


\section{References}

Berkelaar, R. 2011. Risk management during the reconstruction of the underground metrostation Rotterdam Central Station, 3rd International Symposium on Geotechnical Safety and Risk (ISGSR 2011), Vogt, Schuppener, Straub \& Bräu (eds) ISBN 978-3939230-01-4, pp. 643-650.

Brown, G.A., and Tiwari, P. 2010. Using DTS Flow Measurements Below Electrical Submersible Pumps to Optimize Production From Depleted Reservoirs by Changing Injection Support Around the Well, Proc. SPE Annual Technical Conference and Exhibition, Florence, Italy, (19-22 September 2010).

Doornenbal, P., Hopman, V., and Spruit, R. 2011. High resolution monitoring of temperature in diaphragm wall Concrete, 8th International Symposium on Field Measurements in GeoMechanics (FMGM2011), USB-stick

Farouki, O. T. 1981. Thermal properties of soils, United States Army Corps of Engineers Cold Regions Research and Engineering Laboratory Hanover, New Hampshire, USA. p.p. 116

Hwang, R.N., Ishihara, K., and Lee, W.F. 2007. Forensic Studies for Failure in Construction of An Underground Station of the Kaohsiung MRT System; Forensic Geotechnical Engineering October 2009, International Society for Soilmechanics and Geotechnical Engineering TC 40. p.p. $144-148$

Poletto, R.J., and Tamaro, G.J. 2011. Repairs of Diaphragm Walls, Lessons Learned, Proceedings of the 36th Annual Conference on Deep Foundations, Boston MA, USA (DFI 2011), USB-stick

Selker, J. S., Thévenaz, L., Huwald, H., Mallet, A., Luxemburg, W., van de Giesen, N., Stejskal, M., Zeman, J., Westhoff, M. and Parlange, M. B. 2006, Distributed fiber-optic temperature sensing for hydrologic systems, Water Resour. Res., 42, W12202, doi:10.1029/2006WR005326

Sensornet, 2012. Oryx DTS - Data Sheet

Sieler, U., Pabst, R., Neweling, G., and Moormann, C. 2012. Der Einsturz des Stadtarchivs in Köln. Bauliche Maßnahmen zur Bergung der Archivalien und zur Erkundung der Schadensursache. BauPortal (2013) Nr.1, pp. 2-7

Spruit, R., van Tol, A.F., Hopman, V., and Broere, W. 2011. Detecting defects in diaphragm walls prior to excavation, 8th International Symposium on Field Measurements in GeoMechanics (FMGM2011), USB-stick

Spruit, R., van Tol, A.F., Broere, W., Slob, E., and Niederleithinger, E. 2014. Detection of anomalies in diaphragm walls with crosshole sonic logging. Canadian Geotechnical Journal, 2014, 51:369-380, 10.1139/cgj-2013-0204

Thevenaz, L., Nikles, M., Fellay, A., Facchini, M., and Robert, P.A. 1998. Truly distributed strain and temperature sensing using embedded optical fibers, Proc. SPIE 3330, Smart Structures and Materials 1998: Sensory Phenomena and Measurement Instrumentation for Smart Structures and Materials, 301 (July 21, 1998); doi:10.1117/12.316986; http://dx.doi.org/10.1117/12.316986 
Tyler, S.W., Selker, J.S., Hausner, M.B., Hatch, C.E., Torgersen, T., Thodal, C.E., and Schladow, S.G. 2009. Environmental temperature sensing using Raman spectra DTS fiber-optic methods, Water Resources Research, Volume 45, Issue 4, April 2009, DOI: 10.1029/2008WR007052.

Van Tol, A.F., Veenbergen, V., and Maertens, J. 2010. Diaphragm walls, a reliable solution for deep excavations in urban areas? DFI and EFFC, London: Deep Foundation Institute, pp. 335-342.

Van Tol, A.F., and Korff, M. 2012. Deep excavations for Amsterdam metro North-South line: An update and lessons learned, Geotechnical Aspects of Underground Construction in Soft Ground - Proceedings of the 7th International Symposium on Geotechnical Aspects of Underground Construction in Soft Ground, pp. 37-45. 
Figure Captions

Figure 1: Raman scatter principle (after Selker et al. 2006)

Figure 2: Cross section of the ACE-TKF CTC 8xMM cable Figure 3: Temperature adaptation in time

Figure 4: Test setup for determining the response curve

Figure 5: Relative temperature difference for 3 measurement positions (in meters relative to the DTS device) in the linear sensor near the transition zone during the response curve test

Figure 6: Response curve for Sensornet Oryx DTS and single ended ACE-TKF CTC 8xMM fiber based upon ten averaged recordings with acquisition time of 1 minute (model as line and measurements as dots, relative temperature $0.5=50 \%$ of $\delta \mathrm{T}$, where $\delta \mathrm{T}=|\mathrm{T} 1-\mathrm{T} 2|$ and $\mathrm{T} 1=$ constant temperature before transition zone, $\mathrm{T} 2=$ constant temperature after transition zone)

Figure 7: Simplified response curve for Sensornet Oryx DTS and single ended ACE-TKF CTC 8xMM fiber using a $5^{\text {th }}$ order equation. The interface between the two media is located at position $1,5 \mathrm{~m}$.

Figure 8: Measured (dashed) and simulated (continuous) temperature response curves for sensor positions near the transition zone

Figure 9: Cross section with DTS sensors relative to joint and rebar cages as applied during the field tests in Rotterdam

Figure 10: Peak temperature profile for sensor position 2 (Figure 9) during concrete curing with CPT and boring

Figure 11: Expected temperature profile sequence during concrete casting

Figure 12: Subsequent temperature profiles with an interval of 4 minutes in the center of the panel during concrete casting, arrows indicating overlapping temperature profiles of subsequent measurements

Figure 13: Subsequent temperature profiles with an interval of 4 minutes in the joint area during concrete casting, arrows indicating overlapping temperature profiles of subsequent measurements

Figure 14: Subsequent temperature profiles with an interval of 4 minutes in the joint area during slurry refreshing

Figure 15: Measured and simulated temperature profiles in the joint at 19-1-2011 15:58:15. Correlation between measured and simulated graphs $=0.999$ from 10.5 to $20.5 \mathrm{~m}$ below surface level

Figure 16: Measured and simulated temperature profiles in the joint at 19-1-2011 15:58:15, upper interface $0.5 \mathrm{~m}$ too high, lower interface $0.5 \mathrm{~m}$ too low. Correlation between measured and simulated graphs $=0.984$ from 10.5 to $20.5 \mathrm{~m}$ below surface level

Figure 17: Temperature resolution Oryx DTS as a function of sensor length (after Sensornet 2012)

Figure 18: Measured and simulated temperature profiles in the center of the panel 
Figure 19: Position of CSL logs, manual depth recordings and DTS sensors in the panel Figure 20: CSL logs, loss of signal indicating poor concrete

Figure 21: Interpretation of CSL and DTS concrete levels (side view of panel)

Figure 22: Concrete levels recorded in the center and joint of a panel 


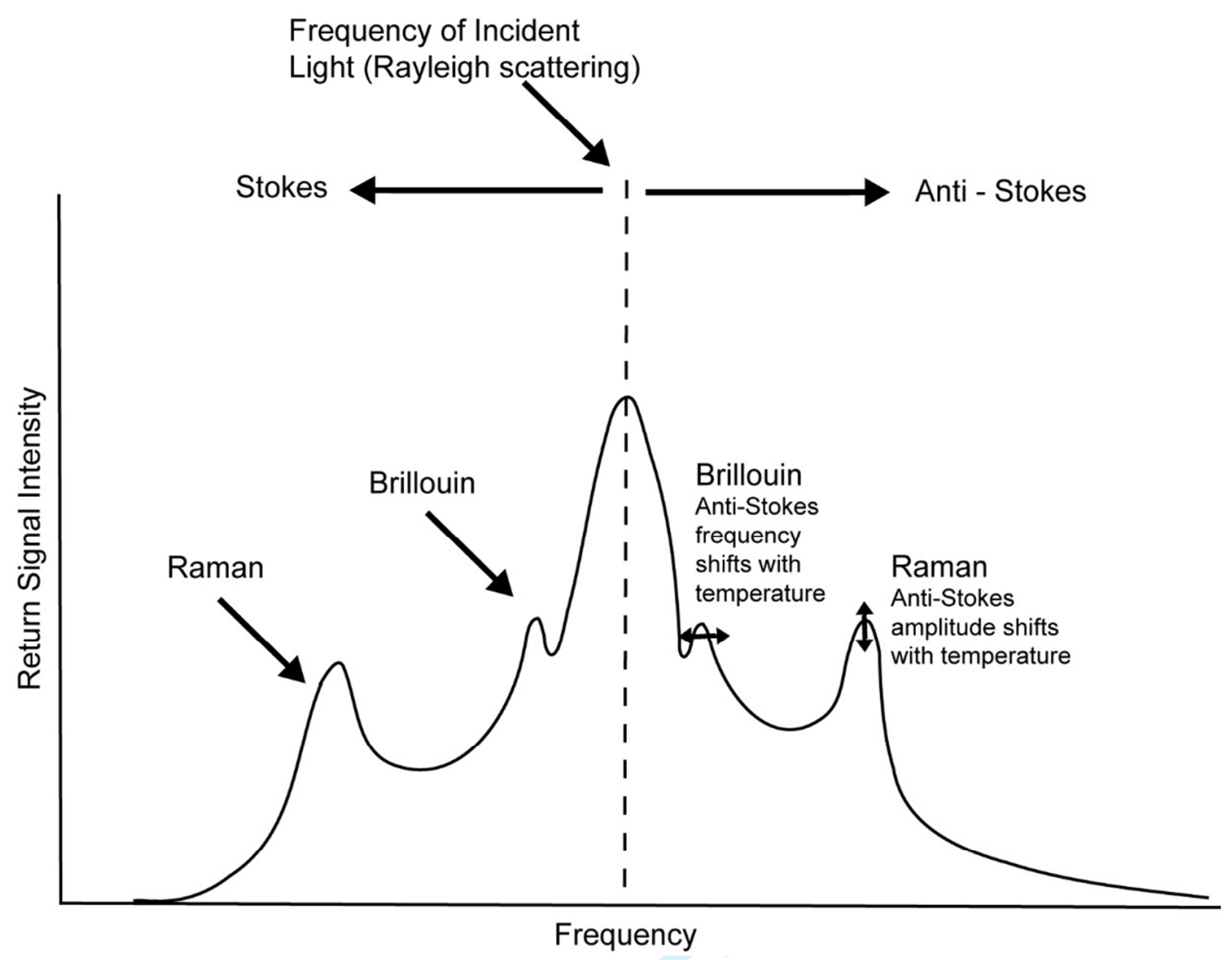

Figure 1: Raman scatter principle (after Selker et al. 2006)

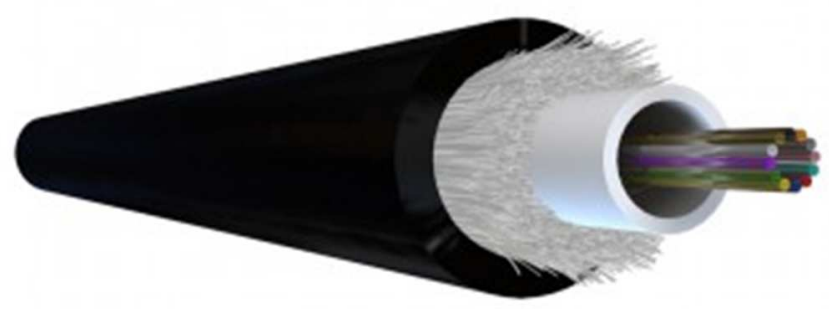

Figure 2: Cross section of the ACE-TKF CTC 8xMM cable 


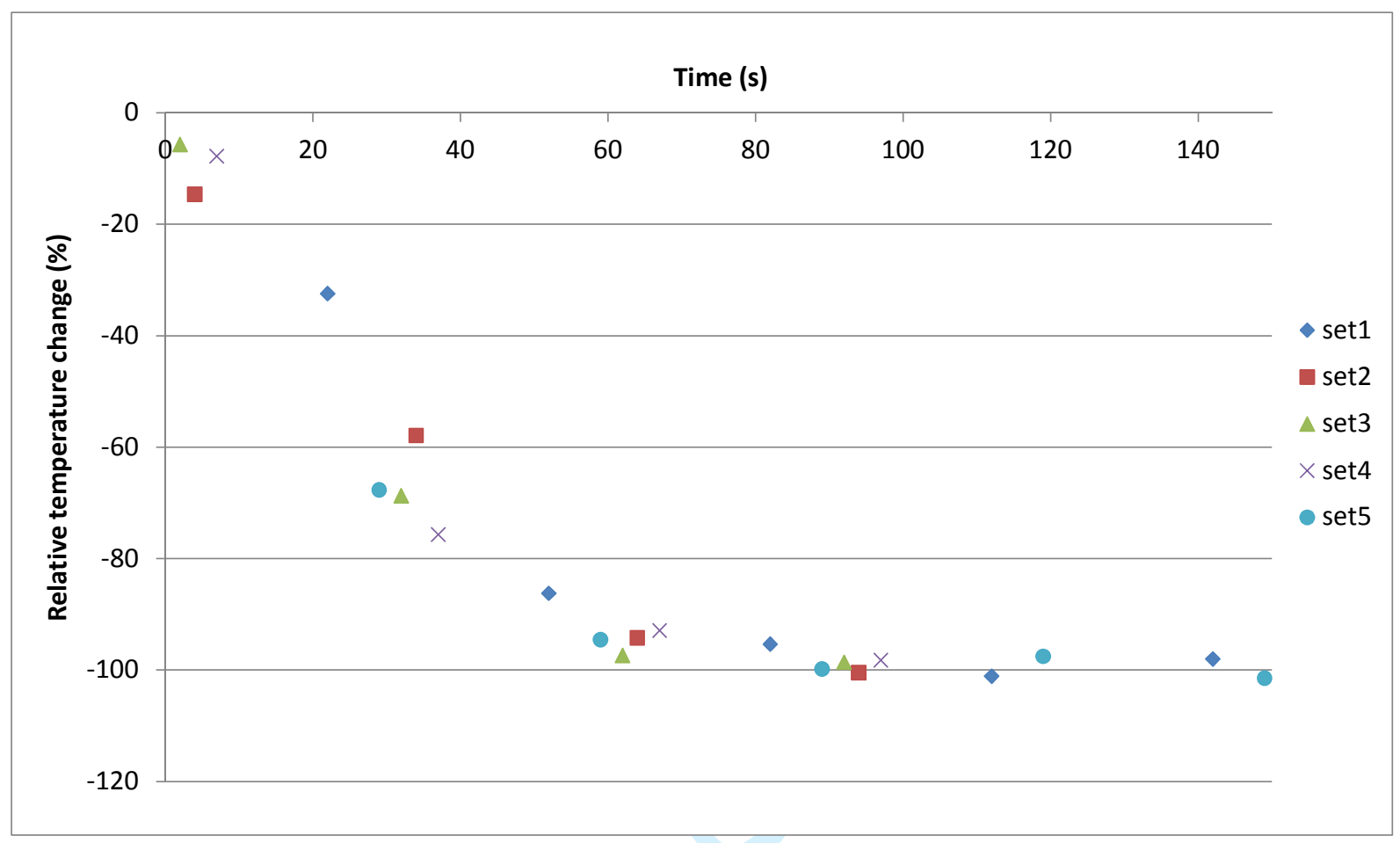

Figure 3: Temperature adaptation in time 


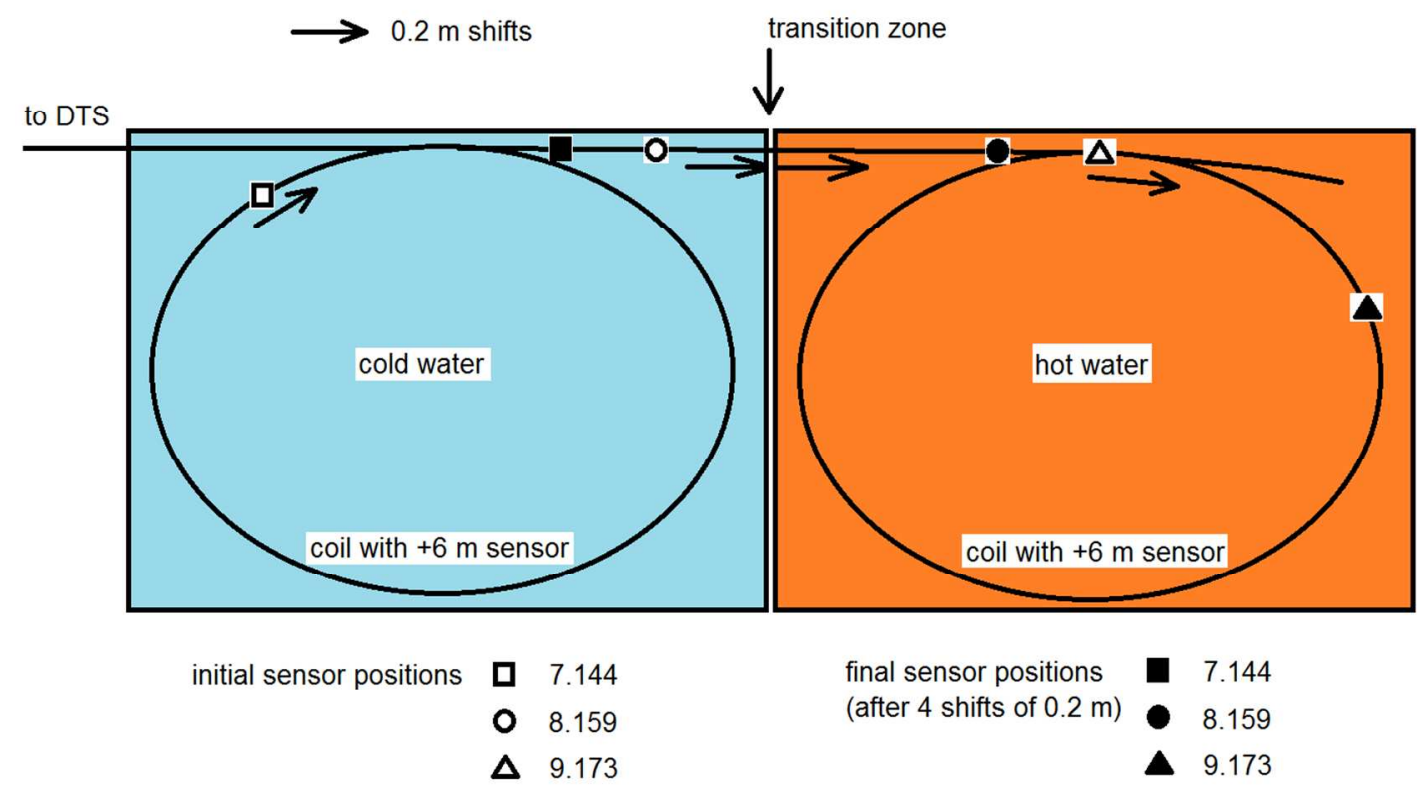

Figure 4: Test setup for determining the response curve

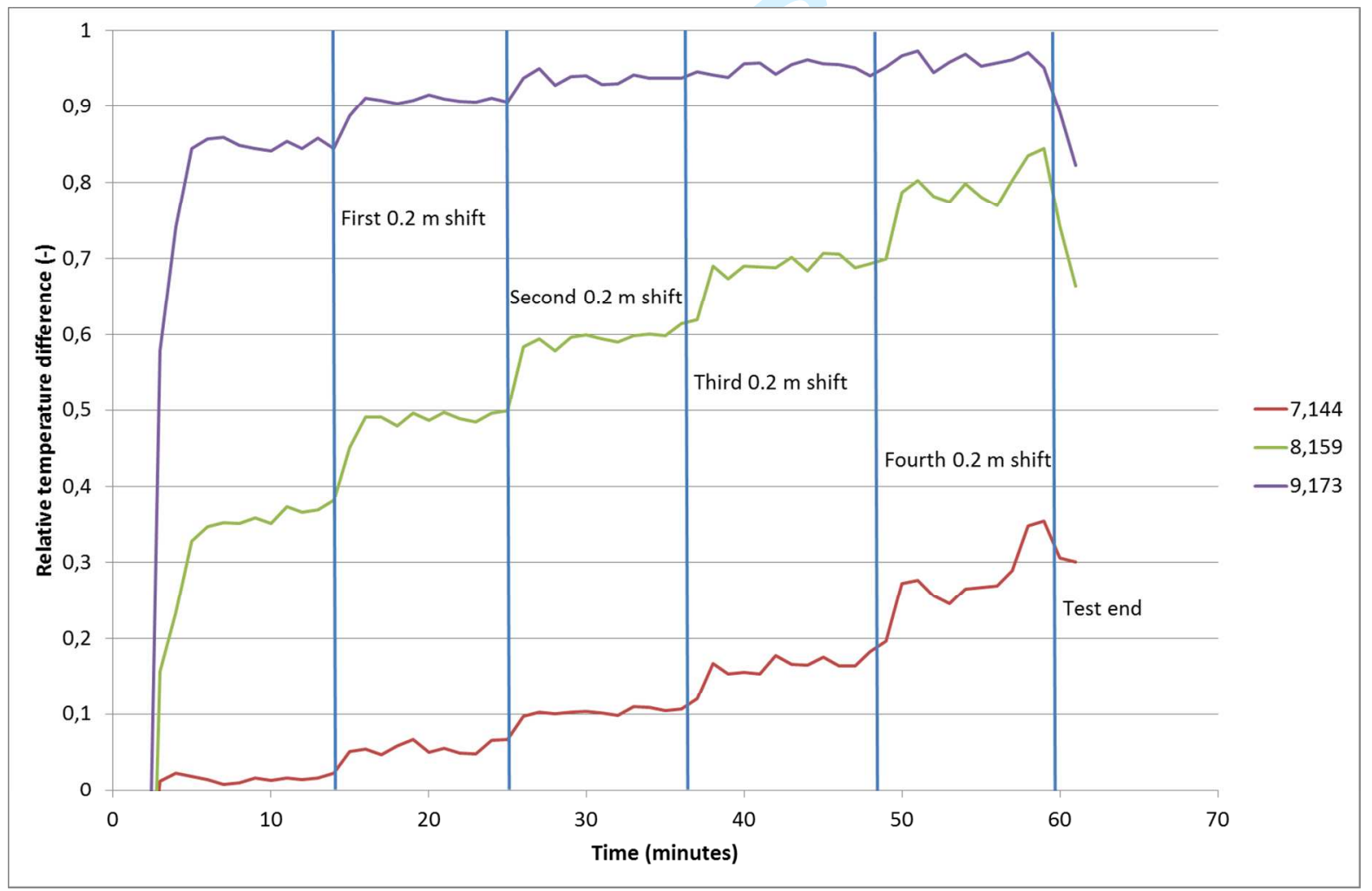

Figure 5: Relative temperature difference for 3 measurement positions (in meters relative to the DTS device) in the linear sensor near the transition zone during the response curve test 


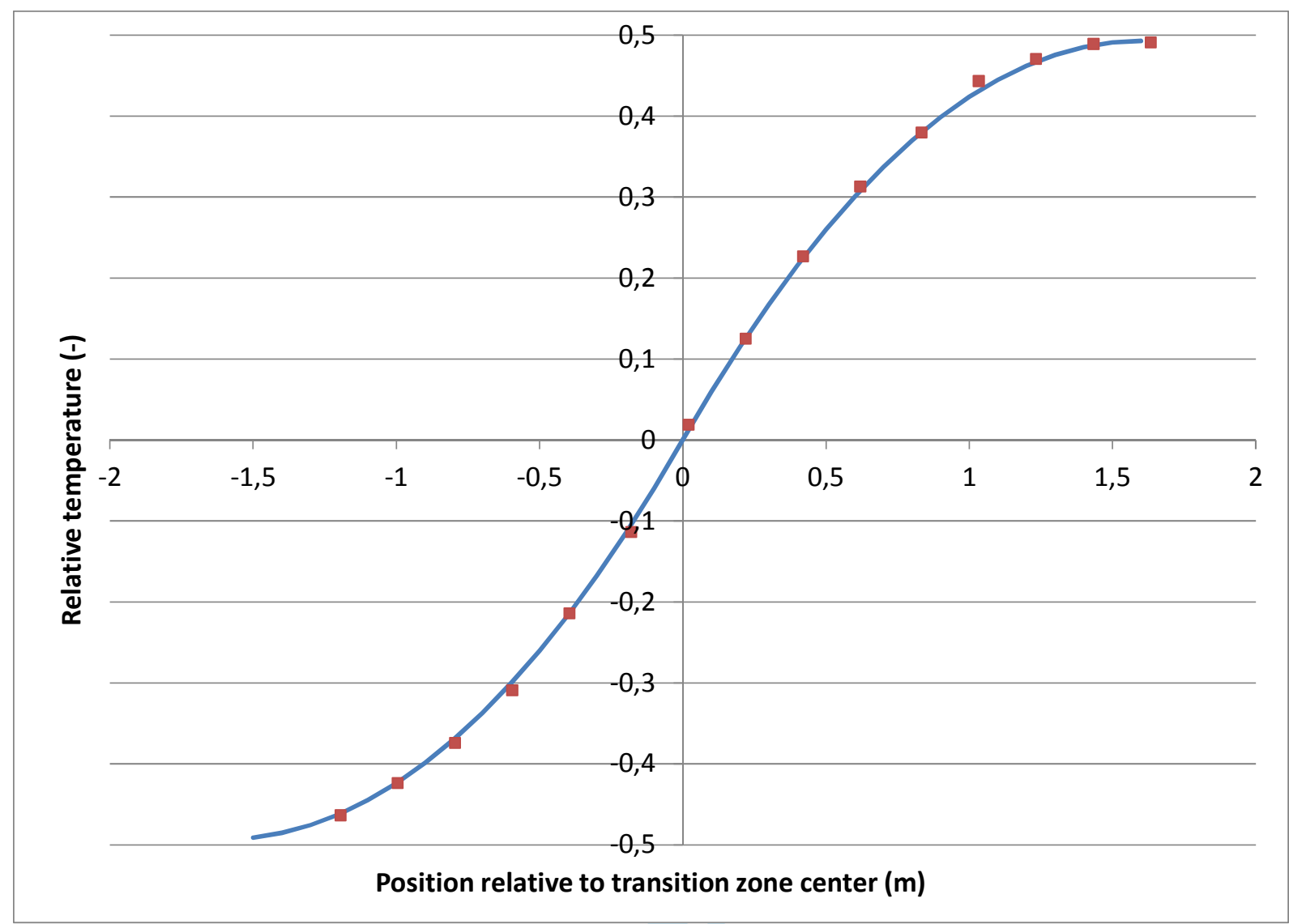

Figure 6: Response curve for Sensornet Oryx DTS and single ended ACE-TKF CTC 8xMM fiber based upon ten averaged recordings with acquisition time of 1 minute (model as line and measurements as dots, relative temperature $0.5=50 \%$ of $\delta \mathrm{T}$, where $\delta \mathrm{T}=|\mathrm{T} 1-\mathrm{T} 2|$ and $\mathrm{T} 1=$ constant temperature before transition zone, $\mathrm{T} 2=$ constant temperature after transition zone) 


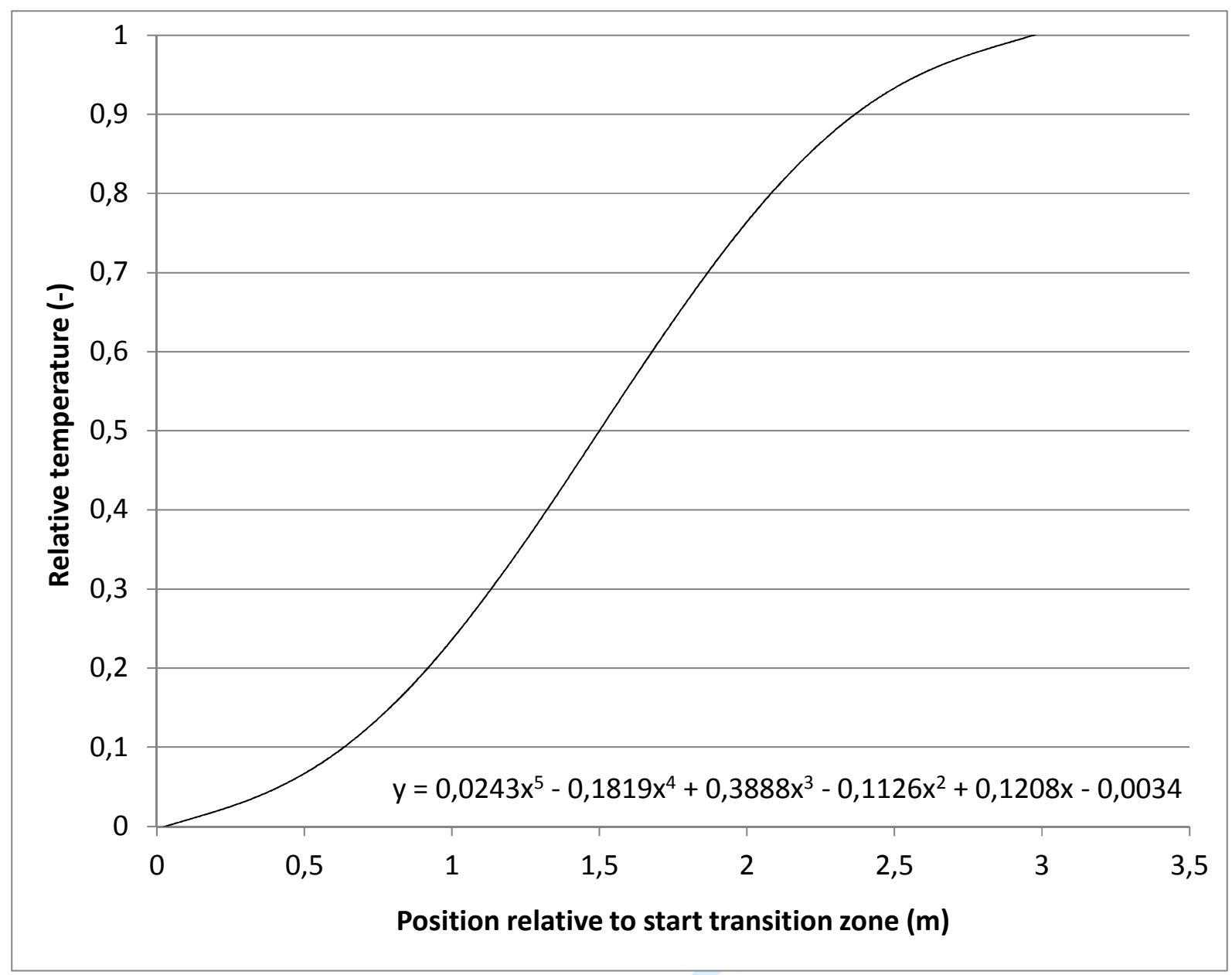

Figure 7: Simplified response curve for Sensornet Oryx DTS and single ended ACE-TKF CTC $8 \mathrm{xMM}$ fiber using a $5^{\text {th }}$ order equation. The interface between the two media is located at position $1,5 \mathrm{~m}$. 


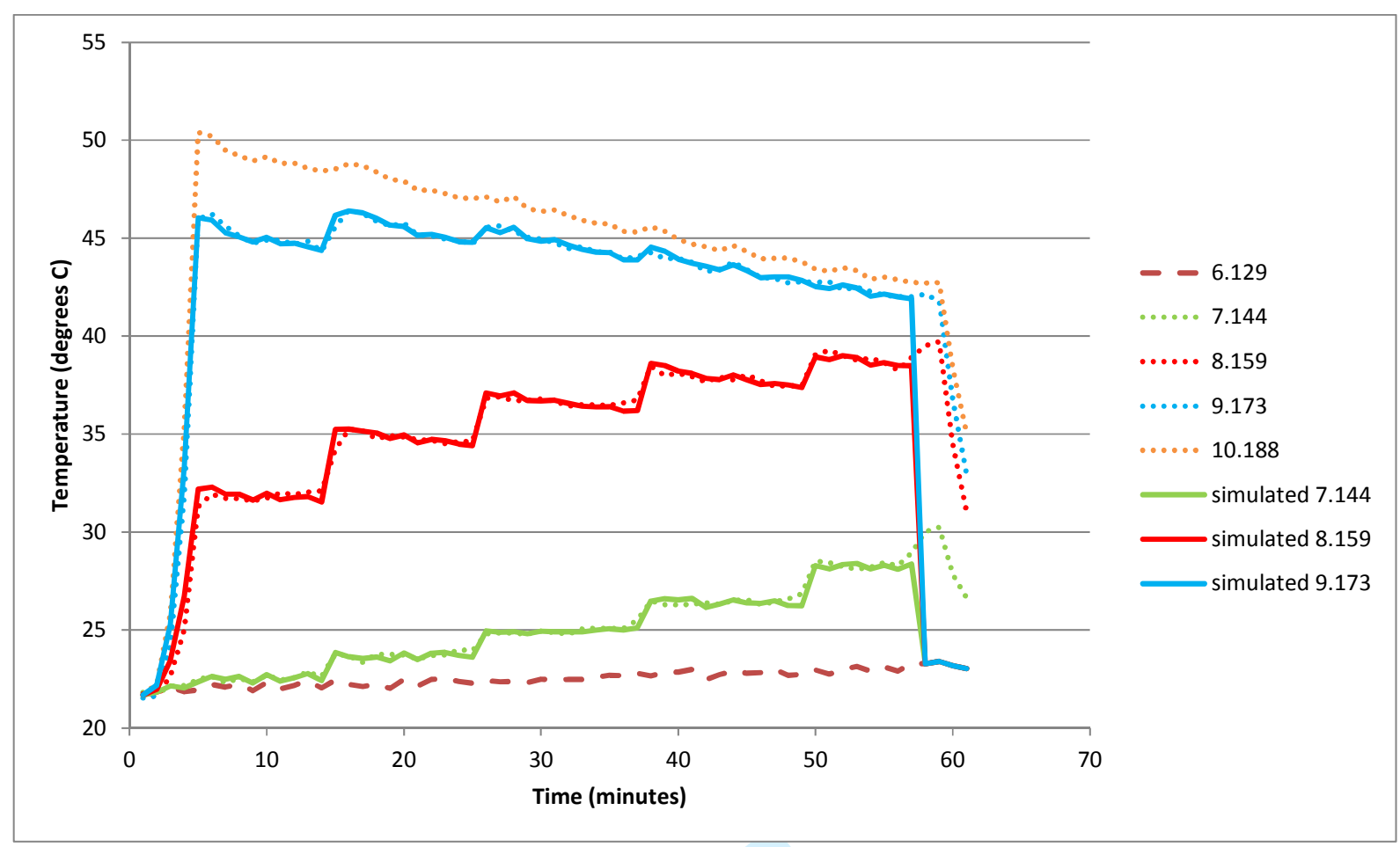

Figure 8: Measured (dashed) and simulated (continuous) temperature response curves for sensor positions near the transition zone

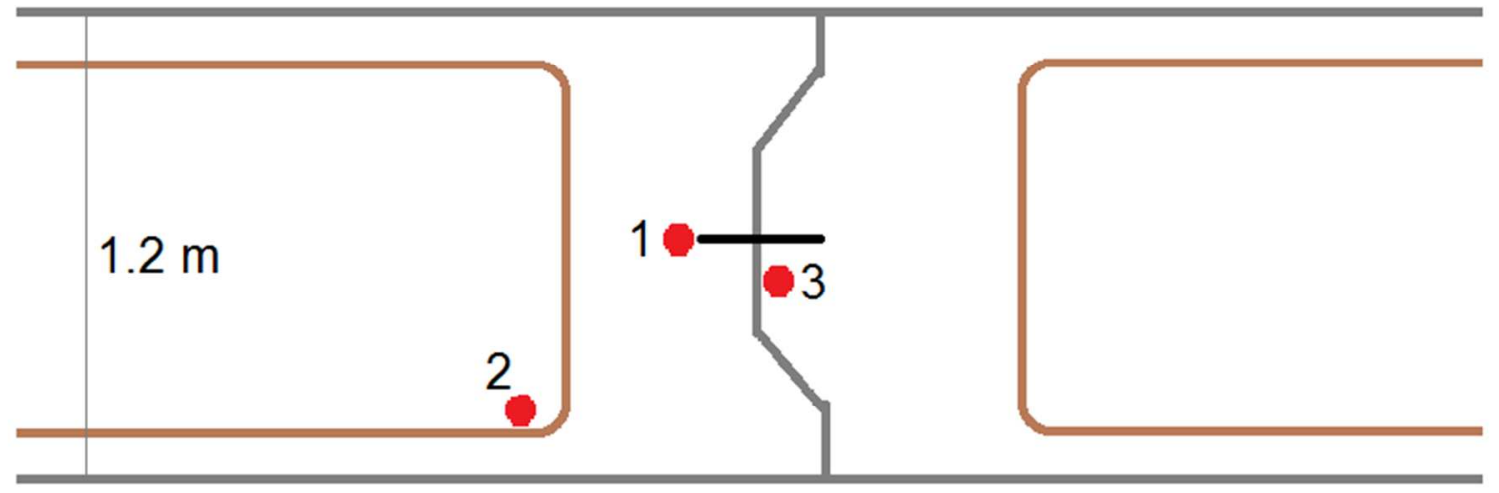

- DTS sensor

Rebar cage

- Rubber water stop

Figure 9: Cross section with DTS sensors relative to joint and rebar cages as applied during the field tests in Rotterdam 


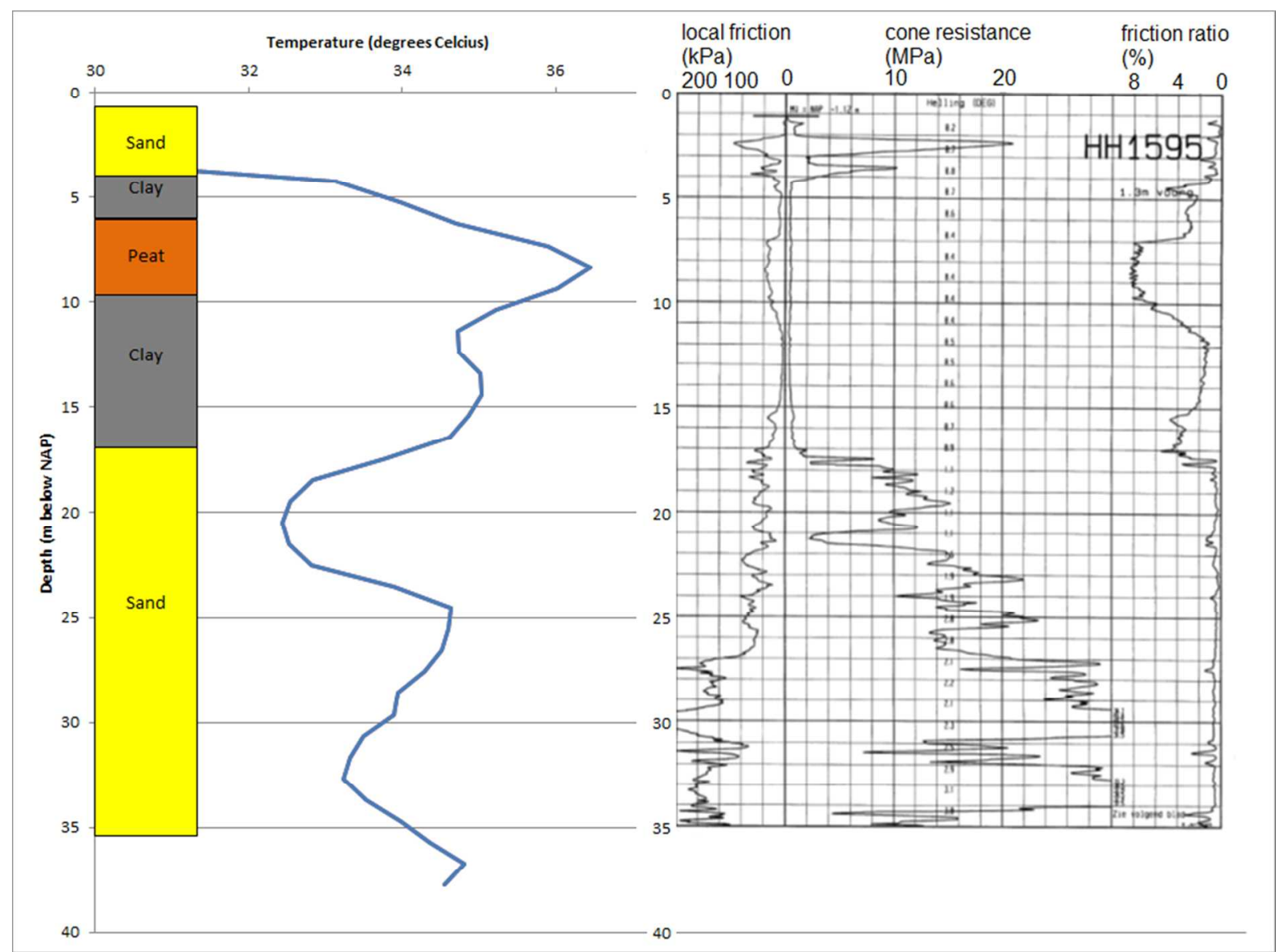

Figure 10: Peak temperature profile for sensor position 2 (Figure 9) during concrete curing with CPT and boring 


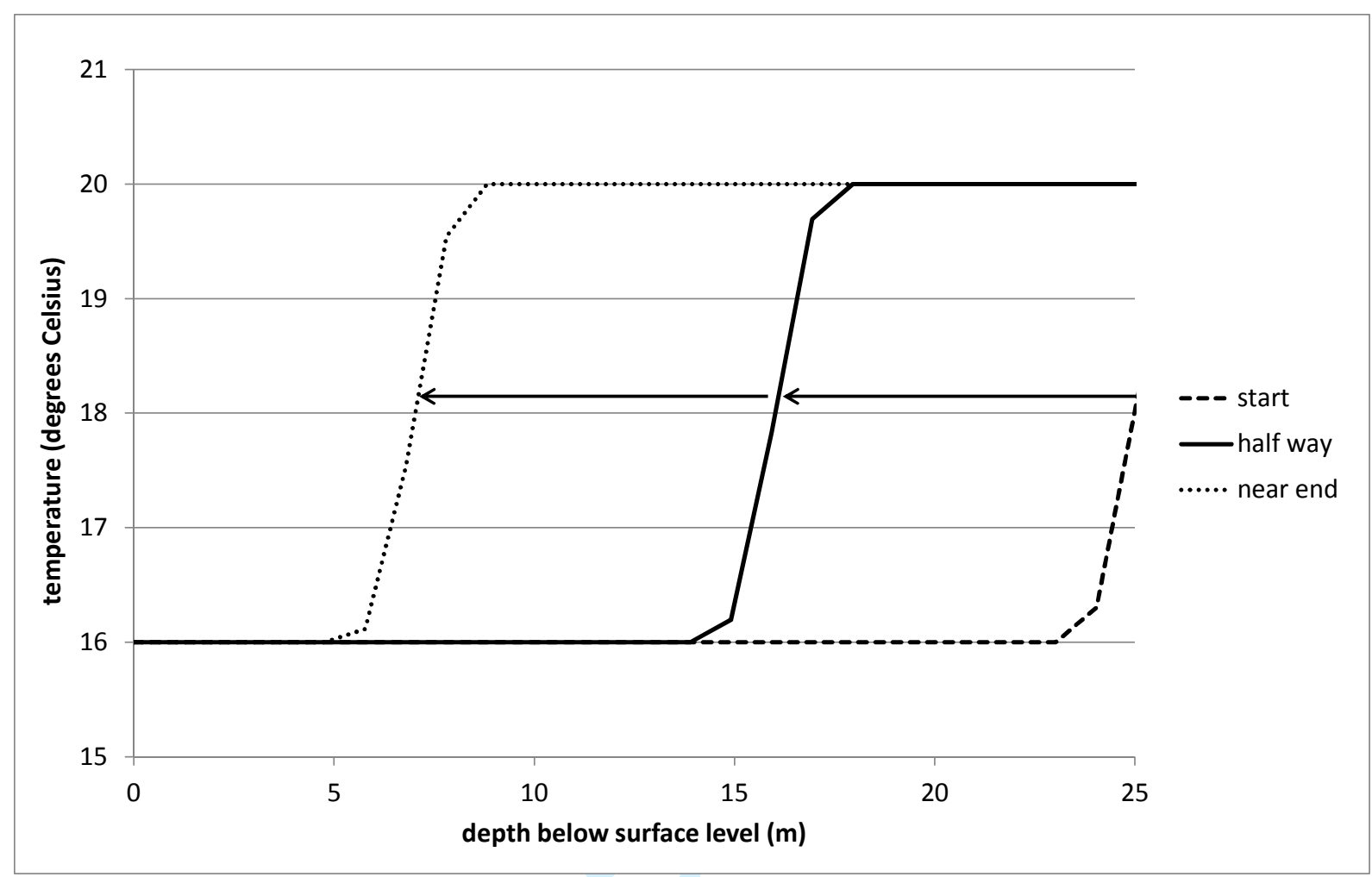

Figure 11: Expected temperature profile sequence during concrete casting 


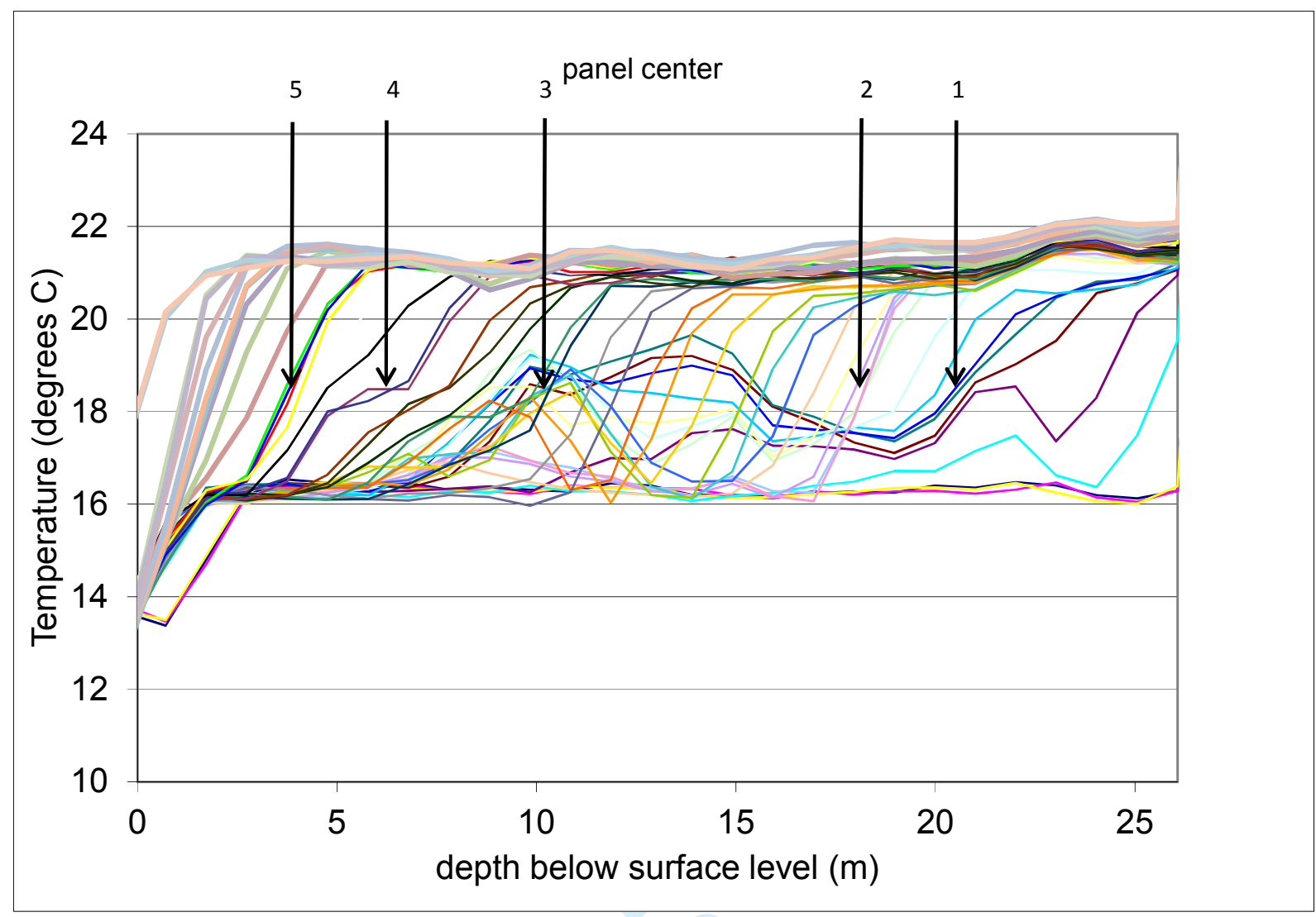

Figure 12: Subsequent temperature profiles with an interval of 4 minutes in the center of the panel during concrete casting, arrows indicating overlapping temperature profiles of subsequent measurements 


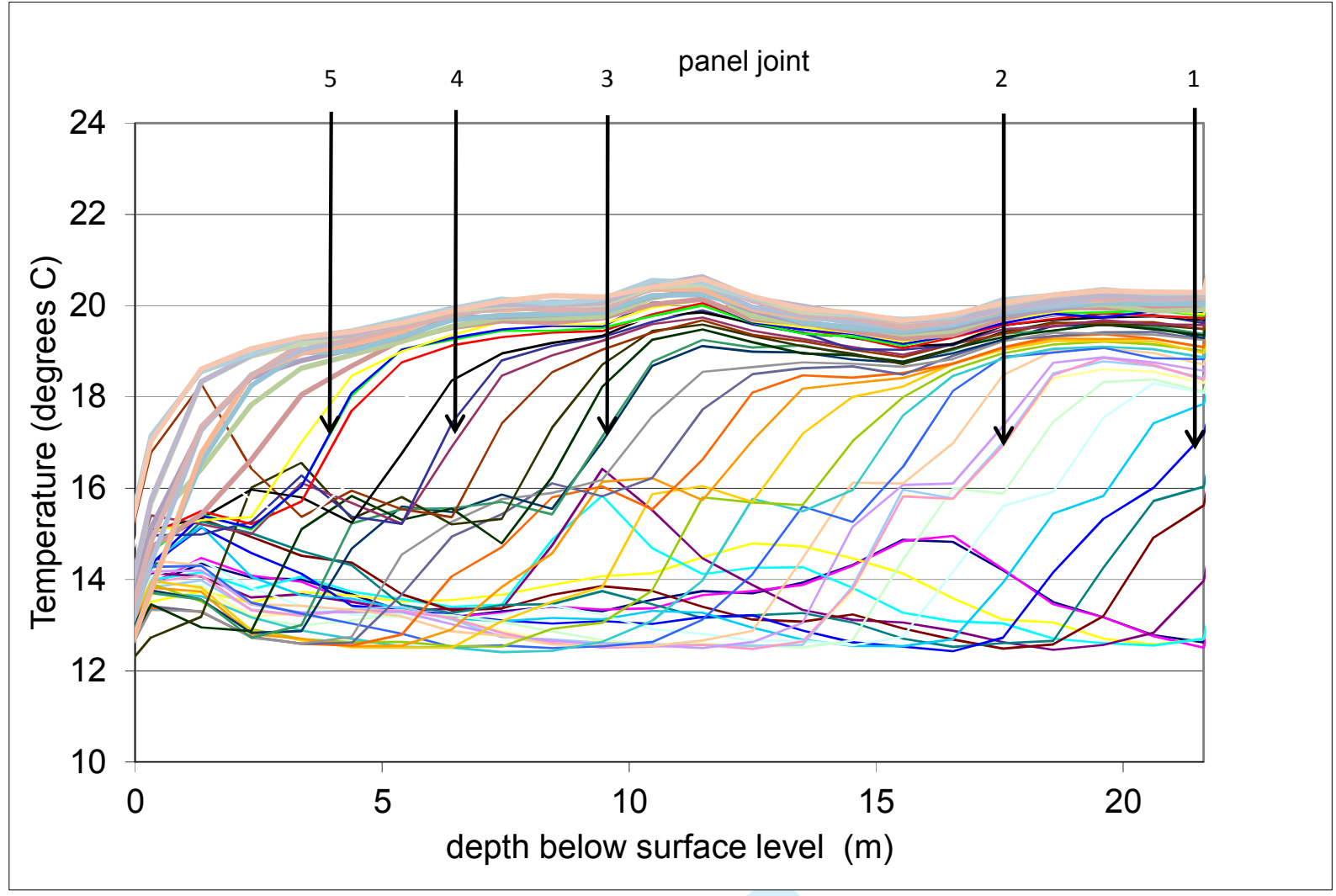

Figure 13: Subsequent temperature profiles with an interval of 4 minutes in the joint area during concrete casting, arrows indicating overlapping temperature profiles of subsequent measurements 


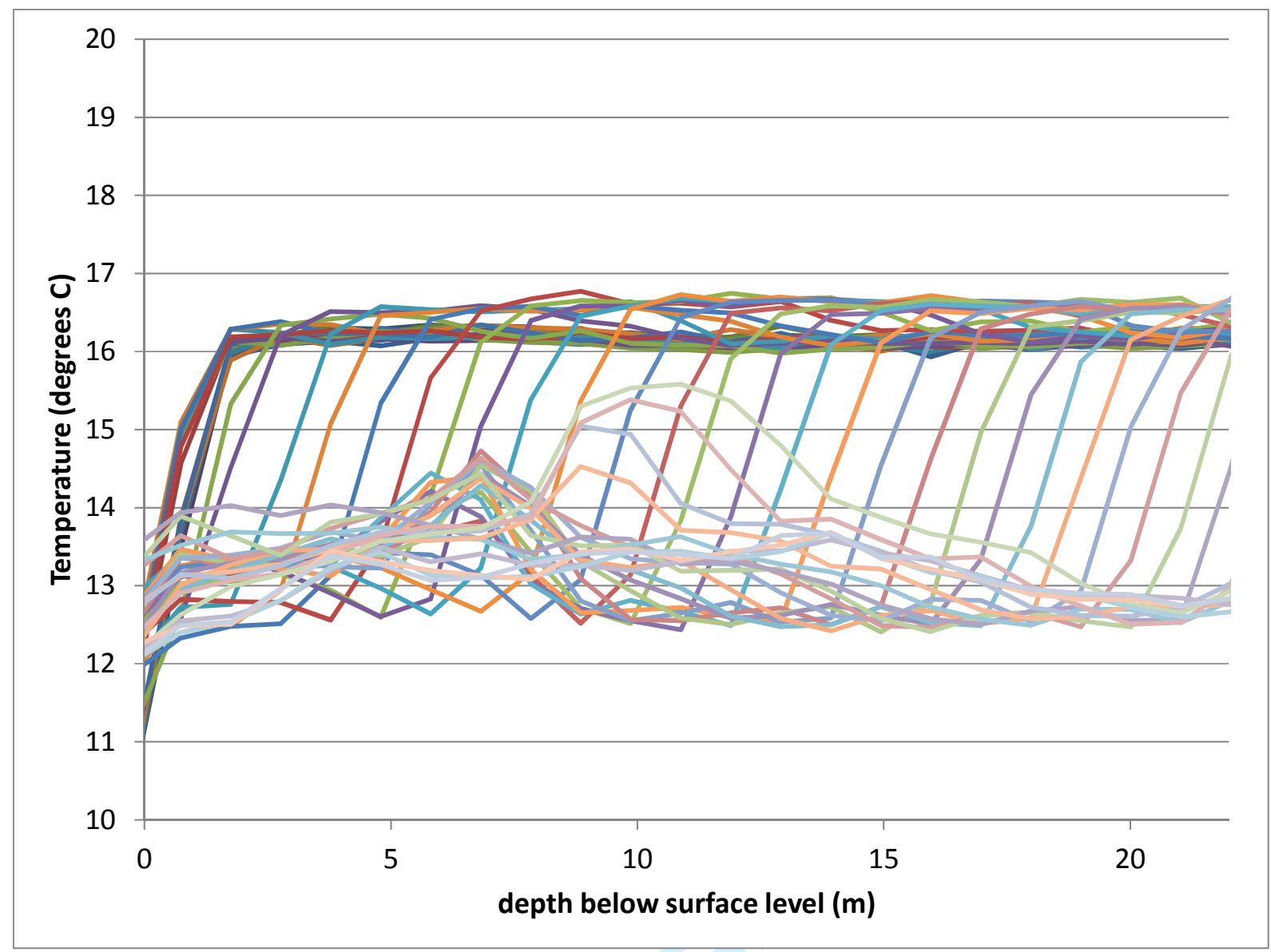

Figure 14: Subsequent temperature profiles with an interval of 4 minutes in the joint area during slurry refreshing 


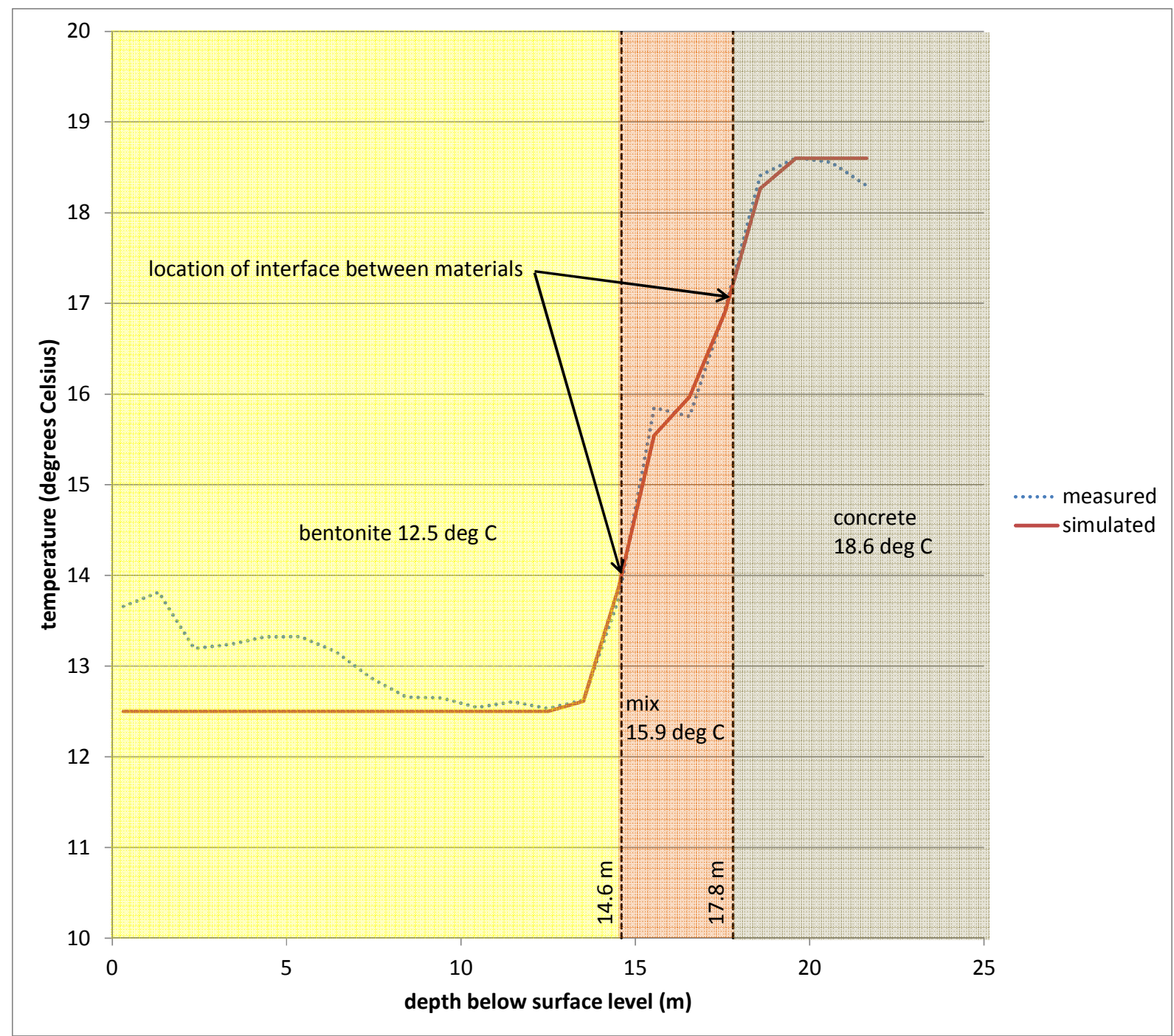

Figure 15: Measured and simulated temperature profiles in the joint at 19-1-2011 15:58:15. Correlation between measured and simulated graphs $=0.999$ from 10.5 to $20.5 \mathrm{~m}$ below surface level 


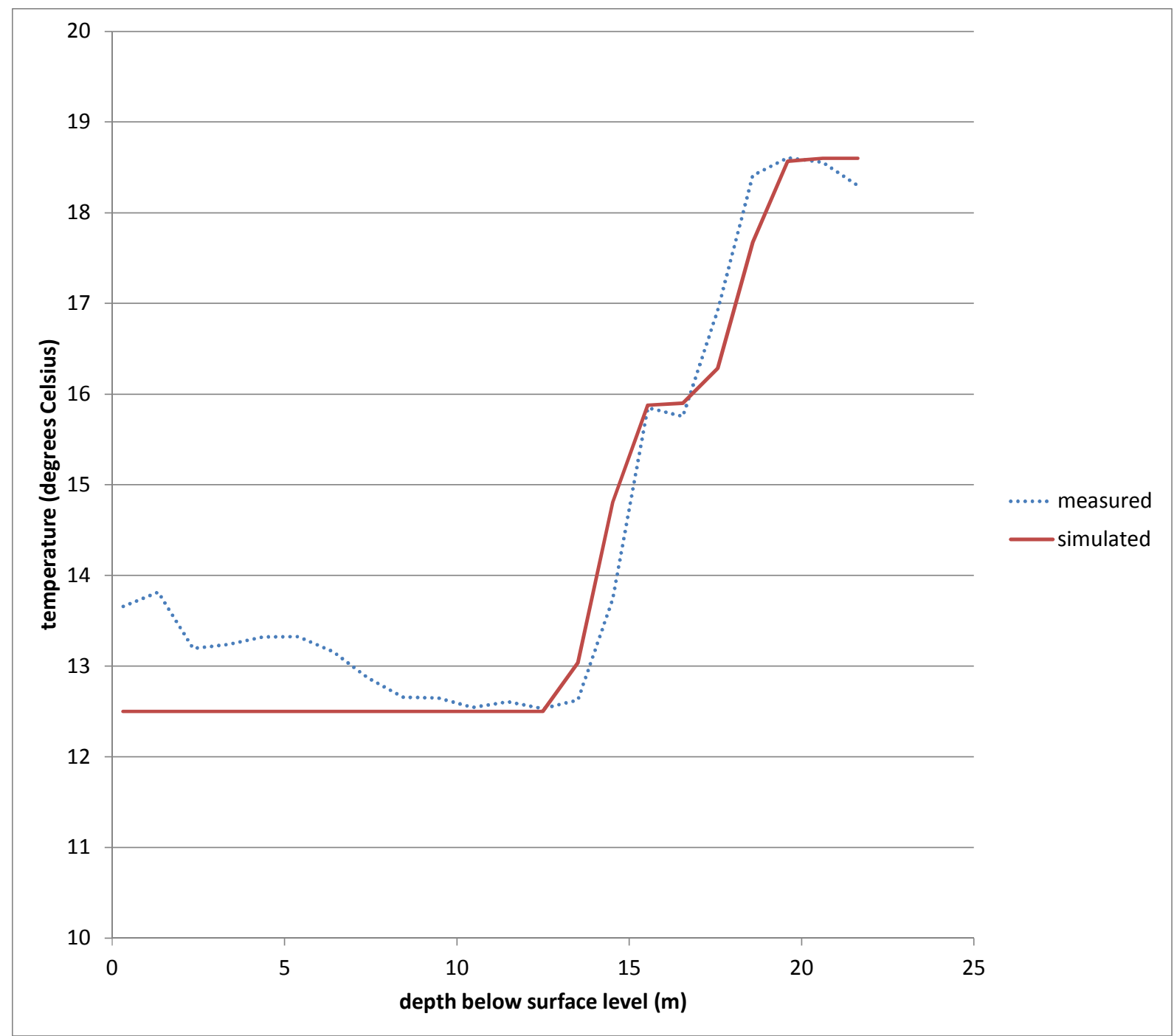

Figure 16: Measured and simulated temperature profiles in the joint at 19-1-2011 15:58:15, upper interface $0.5 \mathrm{~m}$ too high, lower interface $0.5 \mathrm{~m}$ too low. Correlation between measured and simulated graphs $=0.984$ from 10.5 to $20.5 \mathrm{~m}$ below surface level 


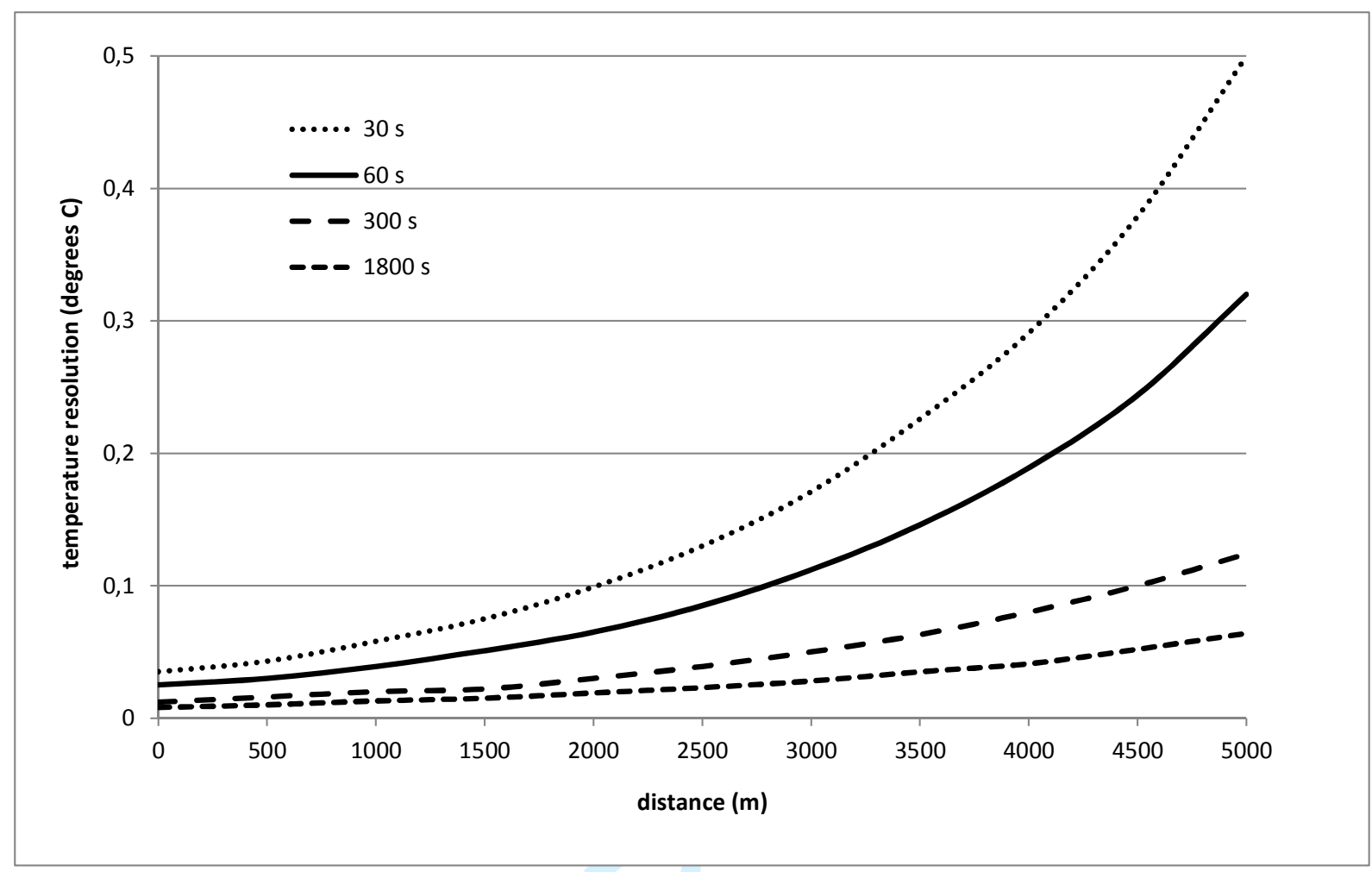

Figure 17: Temperature resolution Oryx DTS as a function of sensor length (after Sensornet 2012) 


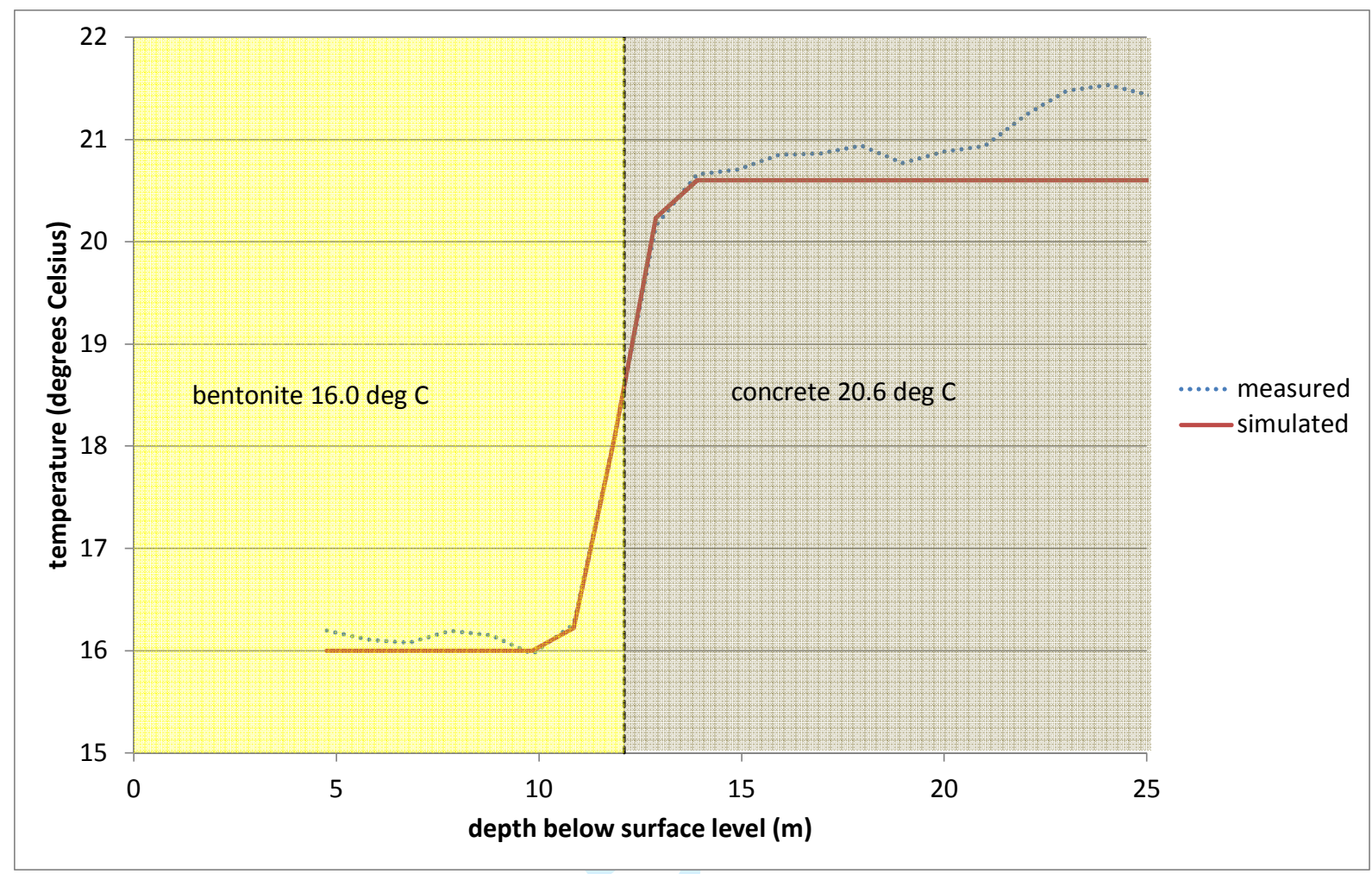

Figure 18: Measured and simulated temperature profiles in the center of the panel

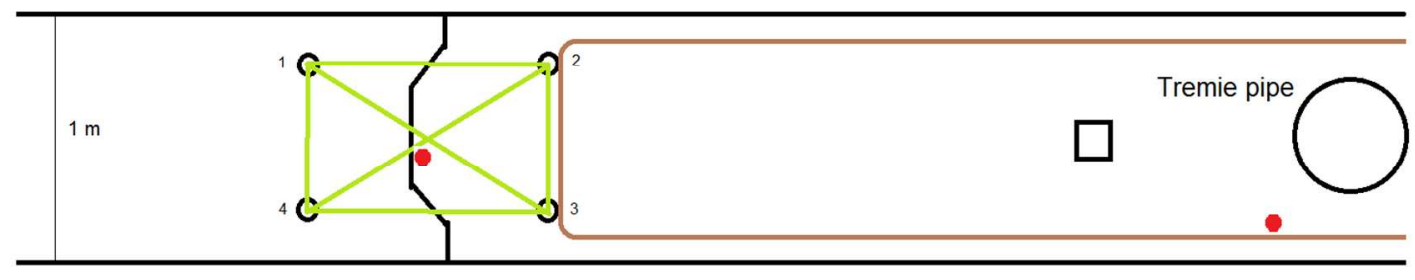

- DTS sensor

- Rebar cage

CSL logs

Manual depth recordings

Figure 19: Position of CSL logs, manual depth recordings and DTS sensors in the panel 


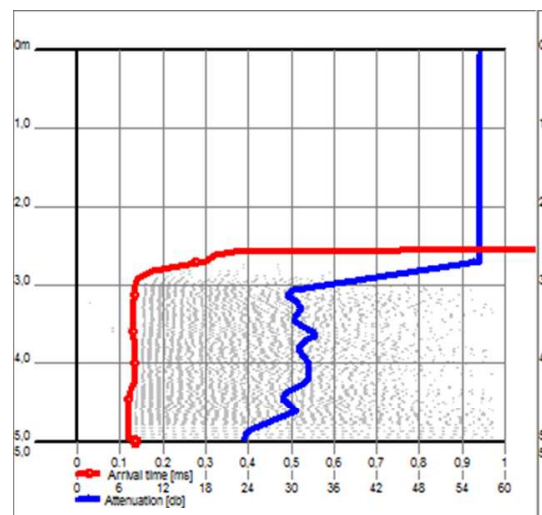

CSL crossing joint straight (3-4)

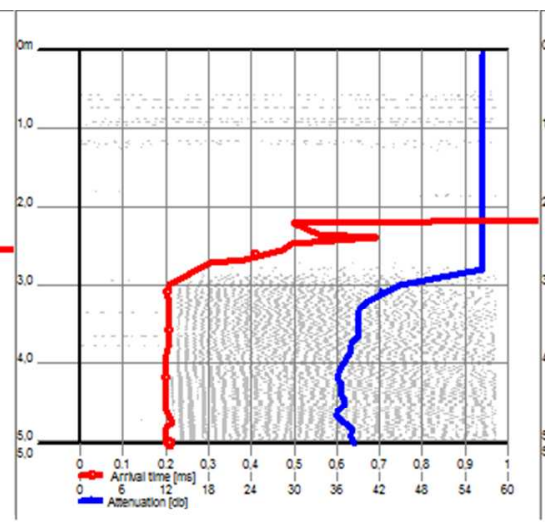

CSL crossing joint diagonally (2-4)

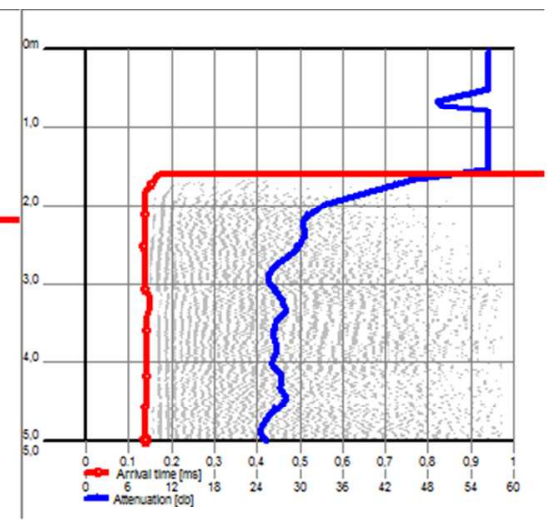

CSL parallel to joint (2-3)

Figure 20: CSL logs, loss of signal indicating poor concrete

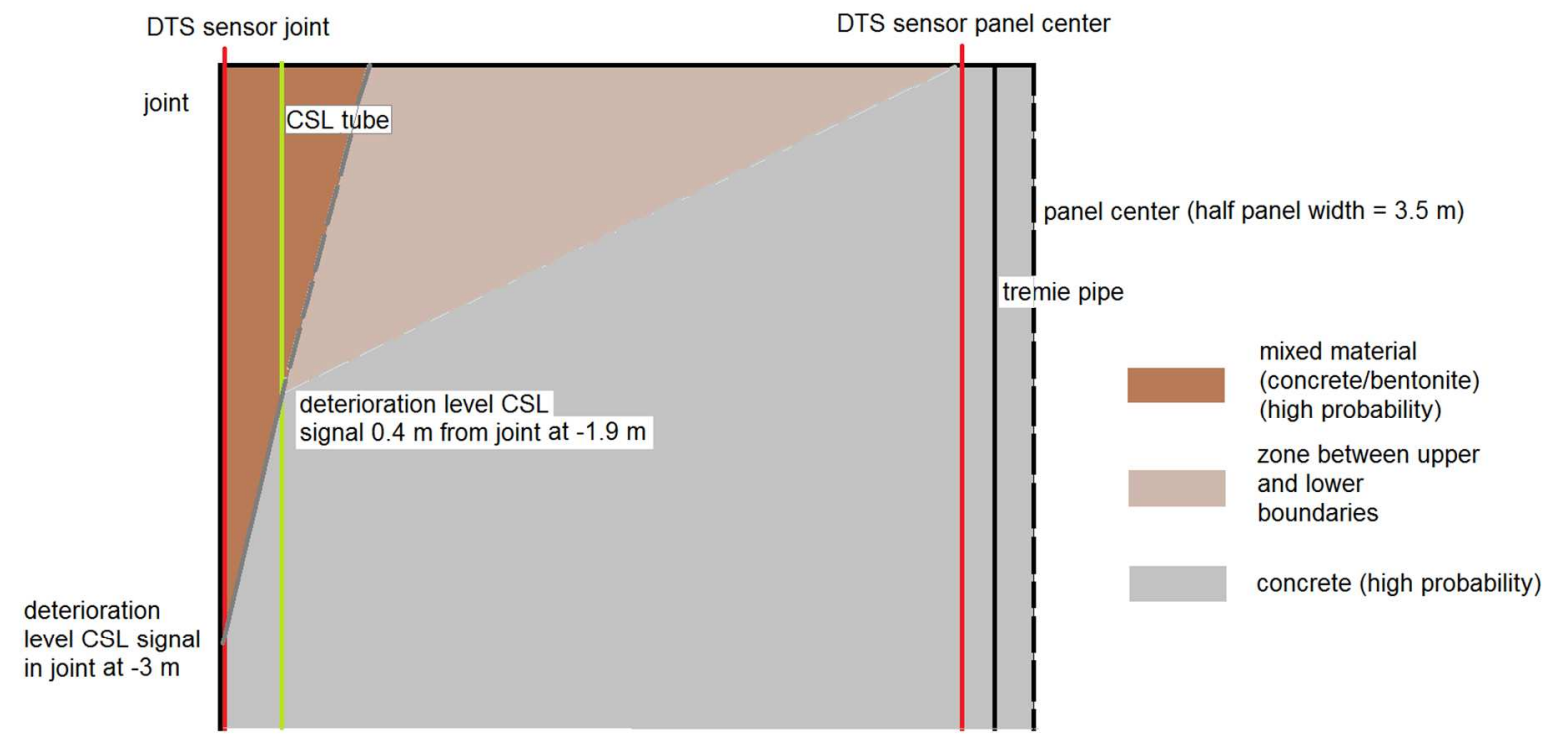

Figure 21: Interpretation of CSL and DTS concrete levels (side view of panel) 


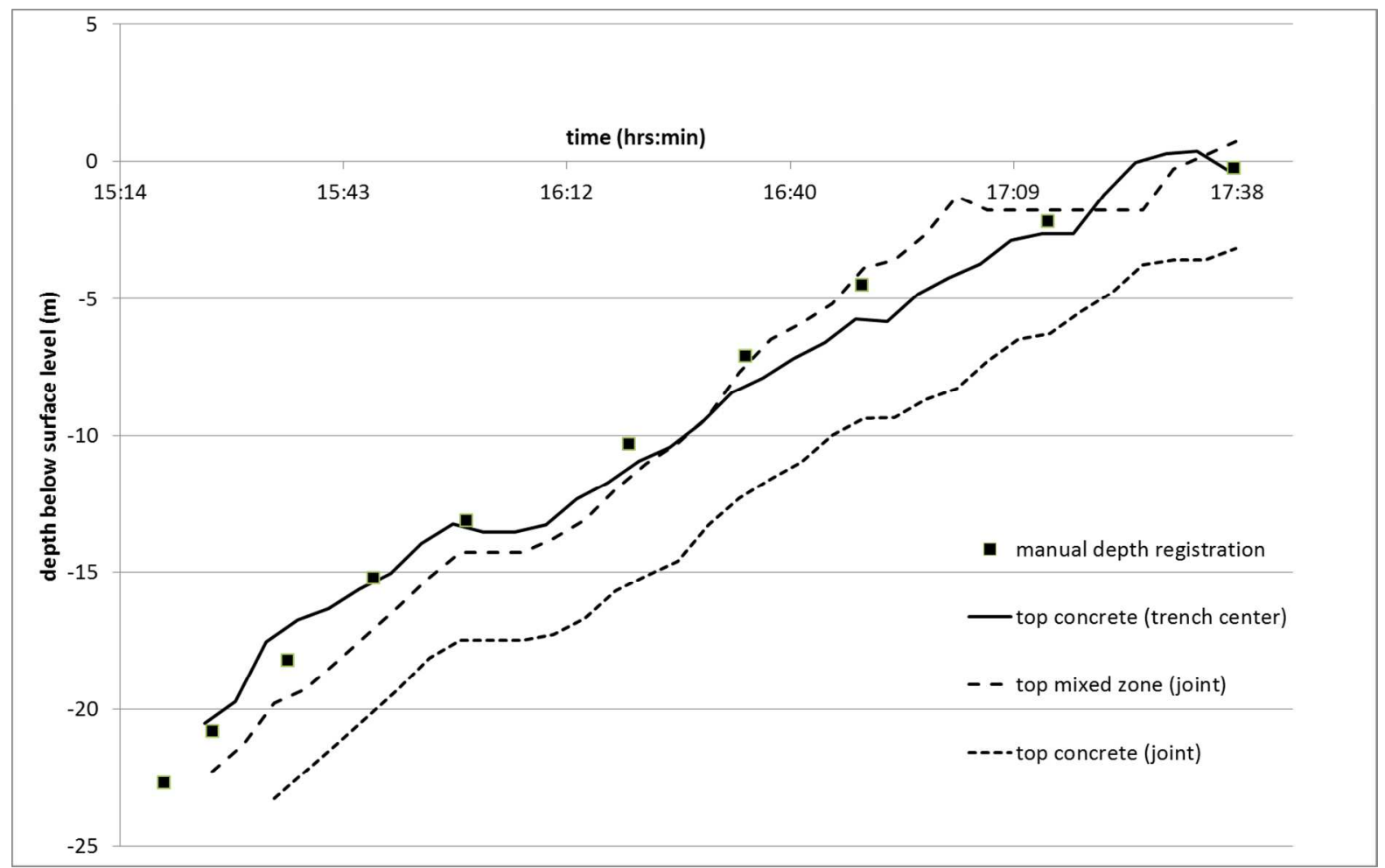

Figure 22: Concrete levels recorded in the center and joint of a panel 